TITLE:

\title{
A rarefied gas flow around a rotating sphere: diverging profiles of gradients of macroscopic quantities
}

\section{$\operatorname{AUTHOR}(\mathrm{S})$ :}

TAGUCHI, Satoshi; SAITO, Kazuyuki; TAKATA, Shigeru

\section{CITATION:}

TAGUCHI, Satoshi ... [et al]. A rarefied gas flow around a rotating sphere: diverging profiles of gradients of macroscopic quantities. Journal of Fluid Mechanics 2019, 862: 5-33

\section{ISSUE DATE:}

2019-03-10

URL:

http://hdl.handle.net/2433/253695

\section{RIGHT:}

This article has been published in a revised form in Journal of Fluid Mechanics http://doi.org/10.1017/ffm.2018.946. This version is free to view and download for private research and study only. Not for re-distribution or re-use. (๑ copyright holder.; This is not the published version. Please cite only the published version.; この論文は出版社版でありません。引 用の際には出版社版をご確認ご利用ください。 


\title{
A rarefied gas flow around a rotating sphere: diverging profiles of gradients of the macroscopic quantities
}

\author{
Satoshi Taguchi ${ }^{1,4} \dagger$, Kazuyuki Saito ${ }^{2}$ and Shigeru Takata ${ }^{3,4}$ \\ ${ }^{1}$ Department of Advanced Mathematical Sciences, Graduate School of Informatics, Kyoto \\ University, Kyoto 606-8501, Japan \\ ${ }^{2}$ Department of Mechanical and Intelligent Systems Engineering, The University of \\ Electro-Communications, Chofu, Tokyo 182-8585, Japan \\ ${ }^{3}$ Department of Aeronautics and Astronautics, Graduate School of Engineering, Kyoto \\ University, Kyoto 615-8540, Japan \\ ${ }^{4}$ Research Project of Fluid Science and Engineering, Advanced Engineering Research Center, \\ Kyoto University
}

(Received xx; revised xx; accepted xx)

The steady behaviour of a rarefied gas around a rotating sphere is studied numerically on the basis of the linearised ellipsoidal statistical model of the Boltzmann equation, also known as the ES model, and the Maxwell diffuse-specular boundary condition. It is demonstrated numerically that the normal derivative of the circumferential component of the flow velocity and that of the heat flux diverge on the boundary with the rate $s^{-1 / 2}$, where $s$ is the normal distance from the boundary. Further, it is demonstrated that the diverging term is proportional to the magnitude of the jump discontinuity of the velocity distribution function on the boundary, which originates from the mismatch of the incoming and outgoing data on the boundary. The moment of force exerted on the sphere is also obtained for a wide range of the Knudsen number and for various values of the accommodation coefficient.

Key words:

\section{Introduction}

In this paper we consider a steady flow of a rarefied gas induced around a rotating sphere in an unbounded domain. The problem is one of the most fundamental external flow problems in fluid mechanics and in rarefied gas dynamics, whose practical applications can be sought in aerosol sciences and/or in vacuum engineering. In this paper, we revisit this classical problem (Loyalka 1992) and carry out precise numerical analysis on the basis of the ellipsoidal statistical model of the Boltzmann equation (the ES model) and the Maxwell diffuse-specular boundary condition.

Our motivation for the present study is twofold. The first motivation is related to the Magnus effect in a rarefied gas. That is, when there is a flow over a rotating sphere, the sphere experiences a lift known as the Magnus force (see e.g. Rubinow \& Keller 1961). Our interest is to understand and clarify the Magnus force acting on a rotating sphere

$\dagger$ Email address for correspondence: taguchi.satoshi.5a@kyoto-u.ac.jp 
in a slow rarefied gas flow, for which the present analysis plays an important role. This will be treated in a forthcoming paper.

The second motivation on which we will focus in the present paper is based on recent theoretical works (Takata \& Taguchi 2017; Takata et al. 2016b) on singular behaviours of the macroscopic quantities of rarefied gases [or the moments of the velocity distribution function (VDF)]. In rarefied gases, there are two main mechanisms underlying the determination of the behaviour of the gas, namely, the molecular (ballistic) transport and collisions (scattering). The effect of the ballistic transport is most highlighted when the gas is in contact with a convex body or boundary. In general, there is a mismatch of the incoming and outgoing data of the unknown (VDF) on a point on the boundary for the molecular velocity tangent to the boundary, if the boundary is convex or flat. In case the boundary is convex, this mismatch, or the discontinuity in VDF, propagates into the gas along the characteristics of the transport equation, causing a singularity in the behaviour of the macroscopic quantities on the boundary (Takata \& Taguchi 2017). More precisely, the normal derivative of the macroscopic quantities diverges in approaching the boundary with diverging rate $s^{-1 / n}$, where $s$ is the normal distance from the boundary and $n(n \geqslant 2)$ is the degree of the dominant terms of the polynomial that locally approximates the boundary.

A spherical body is a typical convex body and the flow around a rotating sphere is subject to this singular behaviour. However, this aspect has not been considered in the preceding study. A further investigation is still necessary in order to clarify the detailed flow features, including not only the flow velocity and the shear stress, but also the heat flow in the gas. In this paper, we will do this numerically. In particular, we will show that the most rapidly diverging term in the macroscopic quantities is proportional to the magnitude of the jump discontinuity of VDF on the boundary. This complements the previous work (Takata \& Taguchi 2017), in which some simplification was needed in the boundary condition to pursue detailed and precise analysis. The Maxwell boundary condition plays an ingenious role for this purpose. We also note that the present issue has a close connection to the S layer (Sone 1973; Sone \& Takata 1992) in the situation where the Knudsen number is small.

Incidentally, when the boundary is of a smooth concave or a plane, the characteristics tangent to the boundary do not enter the gas region. In these cases, a weaker singularity was shown to occur (Takata \& Taguchi 2017; Takata \& Funagane 2011), that is, the normal derivative of the macroscopic quantities diverges in approaching the boundary with diverging rate $\ln s$.

The resting part is organised as follows. After the formulation (section 2), the reduction of the problem is carried out in section 3. Section 4 summarises analytical results for the cases of large and small Knudsen numbers. Section 5 shows the numerical results, followed by discussions (section 6). Section 7 is the conclusion.

\section{Formulation}

\subsection{Problem and basic assumptions}

Let us consider a monatomic ideal gas around a sphere with radius $L$ rotating about a fixed axis passing through the centre with constant angular velocity $\Omega^{*}$. We introduce the space rectangular coordinate system $L x_{i}$ (or $L \boldsymbol{x}$ ) in such a way that the origin is located at the centre of the sphere and that the $x_{1}$ axis is taken to be the axis of revolution of the sphere. At far distance from the sphere, the state of the gas is the resting equilibrium state with density $\rho_{\infty}$, and temperature $T_{\infty}$. The (uniform) temperature of the sphere is 
supposed to be the same as that of the gas at infinity. We investigate the steady behaviour of the gas induced around the sphere, under the following basic assumptions.

(i) The behaviour of the gas is described by the ellipsoidal statistical model (Holway 1966; Andries et al. 2000), which we call the ES model, of the Boltzmann equation.

(ii) The gas molecules are reflected on the sphere surface according to the Maxwell diffuse-specular boundary condition (Sone 2007).

(iii) The angular velocity of the sphere is sufficiently small, i.e. $\left|\Omega^{*}\right| L /\left(2 R T_{\infty}\right)^{1 / 2} \ll 1$, and the equation and boundary condition can be linearised around the reference equilibrium state at rest. Here, $R$ is the specific gas constant (i.e. the Boltzmann constant divided by the mass of a molecule).

We further assume that the state of the sphere surface is homogeneous and therefore the accommodation coefficient of the surface, denoted by $\alpha$, is a constant independent of the position on the surface. In the present study, we assume that the temperature of the sphere is uniform and is the same as that of the gas at infinity. A justification of this assumption is given in Appendix A.

\subsection{Basic equations}

Let us introduce the molecular velocity $\left(2 R T_{\infty}\right)^{1 / 2} \zeta_{i}\left[\right.$ or $\left.\left(2 R T_{\infty}\right)^{1 / 2} \boldsymbol{\zeta}\right]$ and the velocity distribution function (VDF) of the gas molecules $\rho_{\infty}\left(2 R T_{\infty}\right)^{-3 / 2}(1+\phi(\boldsymbol{x}, \boldsymbol{\zeta})) E$, where $E=E\left(\zeta_{i}\right)=\pi^{-3 / 2} \exp \left(-\zeta_{j}^{2}\right)$. We also denote by $\rho_{\infty}(1+\omega(\boldsymbol{x}))$ the density, by $\left(2 R T_{\infty}\right)^{1 / 2} u_{i}(\boldsymbol{x})$ the flow velocity, by $T_{\infty}(1+\tau(\boldsymbol{x}))$ the temperature, by $p_{\infty}(1+P(\boldsymbol{x}))$ the pressures, by $p_{\infty}\left(\delta_{i j}+P_{i j}(\boldsymbol{x})\right)$ the stress tensor and by $p_{\infty}\left(2 R T_{\infty}\right)^{1 / 2} Q_{i}(\boldsymbol{x})$ the heat-flow vector of the gas. Here, $\delta_{i j}$ is the Kronecker delta and $p_{\infty}=R \rho_{\infty} T_{\infty}$. In the sequel, we also use the spherical coordinate system $(\operatorname{Lr}, \theta, \varphi)$ with its polar direction directed to the $x_{1}$ axis. The corresponding components of the molecular velocity are denoted by $\left(2 R T_{\infty}\right)^{1 / 2}\left(\zeta_{r}, \zeta_{\theta}, \zeta_{\varphi}\right)$. The similar convention will be used throughout the paper for vectors and tensors [e.g. $\left(u_{r}, u_{\theta}, u_{\varphi}\right)$ etc].

The linearised ES equation for the present steady problem reads

$$
\begin{aligned}
& \zeta_{j} \frac{\partial \phi}{\partial x_{j}}=\frac{1}{k} \mathcal{L}^{\mathrm{ES}}[\phi], \\
& \mathcal{L}^{\mathrm{ES}}[\phi]=-\phi+\omega+2 \zeta_{j} u_{j}+\left(\zeta_{j}^{2}-\frac{3}{2}\right) \tau+\nu\left(\zeta_{i} \zeta_{j}-\frac{\zeta_{k}^{2}}{3} \delta_{i j}\right) P_{i j}, \\
& \omega=\langle\phi\rangle, \quad u_{i}=\left\langle\zeta_{i} \phi\right\rangle, \quad \tau=\frac{2}{3}\left\langle\left(\zeta_{j}^{2}-\frac{3}{2}\right) \phi\right\rangle, \quad P_{i j}=2\left\langle\zeta_{i} \zeta_{j} \phi\right\rangle,
\end{aligned}
$$

where $\mathcal{L}^{\mathrm{ES}}$ is the linearised collision operator for the ES model with the so-called relaxation parameter $\nu \in[-1 / 2,1)$,

$$
\left\langle g\left(\zeta_{i}\right)\right\rangle=\int g E \mathrm{~d} \boldsymbol{\zeta}
$$

and $k$ is defined by

$$
k=\frac{\sqrt{\pi}}{2} \mathrm{Kn}=\frac{\sqrt{\pi}}{2} \frac{\ell_{\infty}}{L}=\frac{\left(2 R T_{\infty}\right)^{1 / 2}}{A_{c} \rho_{\infty} L} .
$$

Here, $\mathrm{Kn}$ is the Knudsen number with $\ell_{\infty}$ being the mean free path of the gas molecules in the equilibrium state at rest with temperature $T_{\infty}$ and density $\rho_{\infty}$, and $A_{c}$ is a constant such that $A_{c} \rho_{\infty}$ is the collision frequency at the reference state. In (2.4), $\mathrm{d} \boldsymbol{\zeta}=\mathrm{d} \zeta_{1} \mathrm{~d} \zeta_{2} \mathrm{~d} \zeta_{3}$ and the domain of integration is the whole space of $\boldsymbol{\zeta}$.

The Maxwell diffuse-specular boundary condition (or the Maxwell condition for short) 
on the sphere is written as

$$
\begin{aligned}
\phi & =(1-\alpha) \phi\left(x_{i}, \zeta_{i}-2 \zeta_{r} n_{i}\right) \\
& +\alpha\left(-2 \sqrt{\pi} \int_{\zeta_{r}<0} \zeta_{r} \phi E \mathrm{~d} \zeta+2 \Omega \zeta_{\varphi} \sin \theta\right), \quad \zeta_{r}>0, \quad(r=1),
\end{aligned}
$$

where $n_{i}$ is the unit normal vector on the surface of the sphere pointed to the gas, $\zeta_{r}=\zeta_{i} n_{i}, \Omega=\Omega^{*} L /\left(2 R T_{\infty}\right)^{1 / 2}$ and $\alpha \in[0,1]$ is the accommodation coefficient. When $\alpha=1$, the specular-reflection part of the condition (2.6) is absent and the condition is known as the diffuse reflection condition. The Maxwell boundary condition is a model, originally introduced by Maxwell, in which the molecules arriving at the boundary are reflected diffusely with probability $\alpha$ and specularly with probability $1-\alpha$. This model is the well-known gas-surface interaction model which can represent in a simplest way the non-perfect accommodation with the boundary of the reflected molecules. The simple combination of diffuse and specular reflections plays a key role in the subsequent discussions because it allows us to control the magnitude of the discontinuity in VDF on the boundary by changing the parameter $\alpha$ (the specular part produces no discontinuity on the boundary). Other models such as the Cercignani-Lampis model (Cercignani 1988) do not represent the specular boundary and therefore is inadequate for the present purpose of quantifying the relation between the discontinuity and the diverging term in the macroscopic quantities. It is also expected that, though the Maxwell condition is unable to reproduce some physical details of actual molecular scatterings, the global property like the torque acting on the sphere is well represented by this model.

On the other hand, the state of the gas approaches the equilibrium state at rest with density $\rho_{\infty}$ and temperature $T_{\infty}$ (and pressure $p_{\infty}$ ) at infinity. Therefore, we have

$$
\phi \rightarrow 0, \quad(r \rightarrow \infty) \text {. }
$$

The pressure and the heat-flow vector are defined by

$$
P=\frac{2}{3}\left\langle\zeta_{j}^{2} \phi\right\rangle=\omega+\tau, \quad Q_{i}=\left\langle\zeta_{i}\left(\zeta_{j}^{2}-\frac{5}{2}\right) \phi\right\rangle .
$$

If we set $\nu=0$ in the (linearised) ES collision operator (2.2), we obtain the well-known linearised BGK collision operator (Bhatnagar et al. 1954; Welander 1954; Sone 2007):

$$
\begin{aligned}
& \mathcal{L}^{\mathrm{ES}} \rightarrow \mathcal{L}^{\mathrm{BGK}}, \quad(\nu \rightarrow 0), \\
& \mathcal{L}^{\mathrm{BGK}}[\phi]=-\phi+\omega+2 \zeta_{j} u_{j}+\left(\zeta_{j}^{2}-\frac{3}{2}\right) \tau,
\end{aligned}
$$

where $\omega, u_{i}$ and $\tau$ are still defined in (2.3).

When the state of the gas is close to the local equilibrium, the ES model yields the following viscosity $\mu_{\infty}$ and thermal conductivity $\lambda_{\infty}$ in the reference state:

$$
\begin{aligned}
\mu_{\infty} & =\frac{\sqrt{\pi}}{2} \frac{p_{\infty}\left(2 R T_{\infty}\right)^{-1 / 2} \ell_{\infty}}{1-\nu}, \\
\lambda_{\infty} & =\frac{5 \sqrt{\pi}}{4} p_{\infty}\left(2 R T_{\infty}\right)^{-1 / 2} R \ell_{\infty} .
\end{aligned}
$$

Consequently, the corresponding Prandtl number for the ES model, defined by the ratio of the kinematic viscosity to the thermal diffusivity, is given by

$$
\operatorname{Pr}=\frac{5 R}{2} \frac{\mu_{\infty}}{\lambda_{\infty}}=\frac{1}{1-\nu},
$$

which is monotonically increasing in $\nu \in[-1 / 2,1)$. The Prandtl number for a monatomic 
gas is close to $2 / 3$ both experimentally and theoretically (it is exactly $2 / 3$ for the pseudoMaxwell molecules). As seen from (2.13), the ES model yields $\operatorname{Pr}=2 / 3$ by specifying the value $\nu=-1 / 2$. This means that the $\mathrm{ES}$ model has an ability to match both the viscosity and the thermal conductivity simultaneously to experimental data for monatomic gases, while tuning the mean free path. However, this favourable property is less important in the present linearised problem, because as shown below (section 2.3), the temperature of the gas is uniform and therefore the thermal conduction is irrelevant (note that the heat flux does not vanish in the gas though the temperature is uniform). Also for the reason explained in the end of section $5, \nu$ (or $\mathrm{Pr}$ ) is considered as a free parameter and will not be specialised to $\nu=-1 / 2$ ( or $\operatorname{Pr}=2 / 3$ ) in this study.

In the original (nonlinear) ES model, the Boltzmann collision term is replaced by a relaxation operator, which is computationally more tractable. It can be viewed as an extension of the BGK model (for which $\nu=0$ ), and has an advantage over other similar models in that the Boltzmann $\mathrm{H}$ theorem has been proved for $-1 / 2 \leqslant \nu<1$ (Andries et al. 2000). The modification of the original Boltzmann ollision operator may lose some details of the two-body collision mechanics involved in the original collision kernel but retains the important basic properties. Moreover, it has been shown in various flow problems that the adjustment of the mean free path in terms of viscosity or thermal conductivity in accordance with the problem and the quantity under consideration is required to have quantitatively a good agreement between the BGK model and the Boltzmann equation. Various extensions of the ES model have also been proposed in the context of gas mixtures (Brull 2015) and polyatomic gases (Andries et al. 2000).

\subsection{Similarity solution}

The following similarity solution is compatible for the present problem:

$$
\phi=\Omega \zeta_{\varphi} \phi_{S}\left(r, \zeta_{r}, \zeta\right) \sin \theta
$$

where $\zeta=\left(\zeta_{i}^{2}\right)^{1 / 2}=\left(\zeta_{r}^{2}+\zeta_{\theta}^{2}+\zeta_{\varphi}^{2}\right)^{1 / 2}$. With this similarity solution, the problem is reduced to the following spatially one-dimensional problem for $\phi_{S}$, i.e.

$$
\begin{aligned}
& \zeta_{r} \frac{\partial \phi_{S}}{\partial r}+\frac{\zeta^{2}-\zeta_{r}^{2}}{r} \frac{\partial \phi_{S}}{\partial \zeta_{r}}-\frac{\zeta_{r}}{r} \phi_{S}=\frac{1}{k} \mathcal{L}_{1}^{\mathrm{ES}}\left[\phi_{S}\right], \\
& \phi_{S}\left(1, \zeta_{r}, \zeta\right)=(1-\alpha) \phi_{S}\left(1,-\zeta_{r}, \zeta\right)+2 \alpha, \quad \zeta_{r}>0, \\
& \phi_{S} \rightarrow 0, \quad(r \rightarrow \infty),
\end{aligned}
$$

where

$$
\begin{aligned}
& \mathcal{L}^{\mathrm{ES}}\left[\zeta_{\varphi} \phi_{S}\right]=\zeta_{\varphi} \mathcal{L}_{1}^{\mathrm{ES}}\left[\phi_{S}\right]=\zeta_{\varphi}\left(-\phi_{S}+2 \widetilde{u}_{\varphi}+2 \nu \zeta_{r} \widetilde{P}_{r \varphi}\right), \\
& \widetilde{u}_{\varphi}=\frac{1}{2}\left\langle\left(\zeta^{2}-\zeta_{r}^{2}\right) \phi_{S}\right\rangle, \quad \widetilde{P}_{r \varphi}=\left\langle\zeta_{r}\left(\zeta^{2}-\zeta_{r}^{2}\right) \phi_{S}\right\rangle .
\end{aligned}
$$

Substituting (2.14) into (2.3) and (2.8), we find that the macroscopic quantities take the following forms:

$$
\begin{aligned}
& u_{\varphi}=\Omega \widetilde{u}_{\varphi}(r) \sin \theta, \\
& P_{r \varphi}=\Omega \widetilde{P}_{r \varphi}(r) \sin \theta, \\
& Q_{\varphi}=\Omega \widetilde{Q}_{\varphi}(r) \sin \theta
\end{aligned}
$$


and $\omega=u_{r}=u_{\theta}=\tau=P=P_{r r}=P_{r \theta}=P_{\theta \theta}=P_{\theta \varphi}=P_{\varphi \varphi}=Q_{r}=Q_{\theta}=0$. Here, $\widetilde{u}_{\varphi}$ and $\widetilde{P}_{r \varphi}$ are given by (2.19), and

$$
\widetilde{Q}_{\varphi}=\frac{1}{2}\left\langle\left(\zeta^{2}-\zeta_{r}^{2}\right)\left(\zeta^{2}-\frac{5}{2}\right) \phi_{S}\right\rangle
$$

Corresponding to (2.9), we have

$$
\begin{aligned}
& \mathcal{L}_{1}^{\mathrm{ES}} \rightarrow \mathcal{L}_{1}^{\mathrm{BGK}}, \quad(\nu \rightarrow 0), \\
& \mathcal{L}_{1}^{\mathrm{BGK}}\left[\phi_{S}\right]=-\phi_{S}+2 \widetilde{u}_{\varphi} .
\end{aligned}
$$

Multiplying (2.1) by $\zeta_{i} E$ and integrating the result with respect to $\zeta$ yield $\partial P_{i j} / \partial x_{j}=$ 0 , from which one can show $\mathrm{d}\left(r^{3} \widetilde{P}_{r \varphi}\right) / \mathrm{d} r=0$, or equivalently

$$
r^{3} \widetilde{P}_{r \varphi}=\text { const. }
$$

Therefore, $r^{3} \widetilde{P}_{r \varphi}$ is a conserved quantity of the problem. In section 3 , this property plays a crucial role to find a conversion relation between the ES and BGK models (see also, e.g. Cercignani 1988; Takata et al. 2016a, and references therein).

If we denote by $p_{\infty} L^{3}(M, 0,0)$ the moment of force (torque) acting on the sphere, $M$ is given by

$$
M=-\int_{|\boldsymbol{x}|=1} \varepsilon_{1 j k} x_{j} P_{k \ell} n_{\ell} \mathrm{d} S
$$

where $\epsilon_{i j k}$ is the Eddington epsilon and $\mathrm{d} S$ is the surface element on the sphere. By the use of $(2.20 b)$, it is further simplified to

$$
M=-\frac{8}{3} \pi \Omega \widetilde{P}_{r \varphi}(r=1) .
$$

Thus, if we express $M$ as

$$
M=\Omega h_{M},
$$

$h_{M}=h_{M}(k, \operatorname{Pr}, \alpha)$ is given by

$$
h_{M}=-\frac{8}{3} \pi \widetilde{P}_{r \varphi}(r=1)=-\frac{8}{3} \pi\left(r^{3} \widetilde{P}_{r \varphi}(r)\right),
$$

where (2.24) has been used for the second equality. The $h_{M}$ depends not only on $k$ and $\operatorname{Pr}$ (or $\nu$ ), but also on $\alpha$ through the boundary condition; hence, the functional dependency $h_{M}=h_{M}(k, \operatorname{Pr}, \alpha)$. One of our interests is to construct $h_{M}(k, \operatorname{Pr}, \alpha)$ for the ES model for a wide range of the parameter space.

No net force acts on the sphere in the present problem.

In our formulation, the problem has been linearised about the reference equilibrium state at rest under the condition of slow rotation, i.e. $|\Omega|=\left|\Omega^{*}\right| L /\left(2 R T_{\infty}\right)^{1 / 2} \ll 1$. Since the domain is unbounded, it is important to determine the range of $r$ in which the linearisation is valid. In this problem, the perturbed velocity distribution function $\phi$ decays like $r^{-2}$ as $r \rightarrow \infty$ when $k<\infty$. Consequently, the nonlinear term remains smaller than the transport term (i.e. the left-hand side of (2.1)) as $r$ is increased, implying that the linearisation is valid uniformly in the whole gas region. The situation is different from a slow uniform flow past a sphere, for which a matched expansion approach is required to treat the nonlinear effect in the region far from the sphere (Taguchi 2015; Taguchi \& Suzuki 2017). 


\section{Relation between the solutions for the ES model and the BGK model}

The problem contains three parameters $k, \operatorname{Pr}(\nu)$ and $\alpha$. Hence, the necessary amount of computations is quite large. Fortunately, in the case of the ES model, one can express the solution for arbitrary $\nu$ in terms of the solution for $\nu=0$ (the BGK model), thereby reducing the amount of computations. In this section, for the sake of discrimination, we denote the solution of the boundary-value problem (2.15)-(2.17) based on the ES model and that based on the BGK model by $\phi_{S}^{\mathrm{ES}}$ and $\phi_{S}^{\mathrm{BGK}}$, respectively (i.e. $\phi_{S}^{\mathrm{BGK}}=$ $\left.\left.\phi_{S}^{\mathrm{ES}}\right|_{\nu=0}\right)$. Likewise, the corresponding macroscopic quantities are distinguished using the superscript ES or BGK.

Supposing that $\phi_{S}^{\mathrm{BGK}}$ is known, we seek $\phi_{S}^{\mathrm{ES}}$ in the form

$$
\phi_{S}^{\mathrm{ES}}=a(r)+b \phi_{S}^{\mathrm{BGK}},
$$

where $a$ is a function of $r$ and $b$ is independent of $\left(r, \zeta_{r}, \zeta\right)$. Thanks to the linearity of $\mathcal{L}_{1}^{\mathrm{ES}}$ and $\mathcal{L}_{1}^{\mathrm{ES}}[1]=0$, we deduce

$$
\mathcal{L}_{1}^{\mathrm{ES}}\left[\phi_{S}^{\mathrm{ES}}\right]-b \mathcal{L}_{1}^{\mathrm{BGK}}\left[\phi_{S}^{\mathrm{BGK}}\right]=\mathcal{L}_{1}^{\mathrm{ES}}\left[\phi_{S}^{\mathrm{ES}}-b \phi_{S}^{\mathrm{BGK}}\right]+2 b \nu \zeta_{r} \widetilde{P}_{r \varphi}^{\mathrm{BGK}}=2 b \nu \zeta_{r} \widetilde{P}_{r \varphi}^{\mathrm{BGK}}
$$

Thus, the subtraction of (2.15) with $\phi_{S}=\phi_{S}^{\mathrm{ES}}$ and that with $\phi_{S}=\phi_{S}^{\mathrm{BGK}}$ (multiplied by b) leads to

$$
\zeta_{r} \frac{\partial\left(\phi_{S}^{\mathrm{ES}}-b \phi_{S}^{\mathrm{BGK}}\right)}{\partial r}-\frac{\zeta_{r}}{r}\left(\phi_{S}^{\mathrm{ES}}-b \phi_{S}^{\mathrm{BGK}}\right)=\frac{2 \nu b}{k} \zeta_{r} \widetilde{P}_{r \varphi}^{\mathrm{BGK}},
$$

or equivalently,

$$
r \frac{\mathrm{d}}{\mathrm{d} r}\left(\frac{a}{r}\right)=\frac{2 \nu b}{k} \widetilde{P}_{r \varphi}^{\mathrm{BGK}}(r) .
$$

On the other hand, (2.24) allows one to write

$$
\widetilde{P}_{r \varphi}^{\mathrm{BGK}}=\frac{\left.\widetilde{P}_{r \varphi}^{\mathrm{BGK}}\right|_{r=1}}{r^{3}} .
$$

Substituting this into (3.2) and integrating the result with respect to $r$ yield

$$
a=-\frac{2}{3} \frac{\nu b}{k} \frac{\left.\widetilde{P}_{r \varphi}^{\mathrm{BGK}}\right|_{r=1}}{r^{2}}+\beta r,
$$

where $\beta$ is an integration constant.

The constants $\beta$ and $b$ are determined as follows. First, condition (2.17) at infinity requires $a \rightarrow 0$ as $r \rightarrow \infty$, and hence $\beta=0$. Next, after noting that both $\phi_{S}^{\mathrm{ES}}$ and $\phi_{S}^{\mathrm{BGK}}$ satisfy the boundary condition (2.16) at $r=1$ independently (for the same $\alpha>0$ ), we have

$$
a(1)=2-2 b \text {. }
$$

Applying this to (3.4) (with $\beta=0$ ) determines $b$, and hence $a(r)$, as follows:

$$
\begin{aligned}
a & =-\frac{2 \nu}{3 k} \frac{\left.\widetilde{P}_{r \varphi}^{\mathrm{BGK}}\right|_{r=1}}{1-\left.(\nu / 3 k) \widetilde{P}_{r \varphi}^{\mathrm{BGK}}\right|_{r=1}} \frac{1}{r^{2}}, \\
b & =\frac{1}{1-\left.(\nu / 3 k) \widetilde{P}_{r \varphi}^{\mathrm{BGK}}\right|_{r=1}} .
\end{aligned}
$$


To summarise, $\phi_{S}^{\mathrm{ES}}$ is expressed in terms of $\phi_{S}^{\mathrm{BGK}}$ as

$$
\phi_{S}^{\mathrm{ES}}=\left(1+\frac{1}{8 \pi} \frac{\operatorname{Pr}-1}{\operatorname{Pr} k} h_{M}^{\mathrm{BGK}}\right)^{-1}\left(\phi_{S}^{\mathrm{BGK}}+\frac{1}{4 \pi} \frac{\operatorname{Pr}-1}{\operatorname{Pr} k} \frac{h_{M}^{\mathrm{BGK}}}{r^{2}}\right),
$$

where we have replaced $\left.\widetilde{P}_{r \varphi}^{\mathrm{BGK}}\right|_{r=1}$ and $\nu$ by $h_{M}^{\mathrm{BGK}}\left(\equiv h_{M}(k, 1, \alpha)\right)$ and Pr, respectively, by the use of the relation $h_{M}^{\mathrm{BGK}}=-\left.(8 / 3) \pi \widetilde{P}_{r \varphi}^{\mathrm{BGK}}\right|_{r=1}$ and (2.13). The corresponding formulas for the macroscopic variables (i.e. the moments of $\phi_{S}$ ) are summarised as

$$
\begin{aligned}
& \widetilde{u}_{\varphi}^{\mathrm{ES}}=\left(1+\frac{1}{8 \pi} \frac{\operatorname{Pr}-1}{\operatorname{Pr} k} h_{M}^{\mathrm{BGK}}\right)^{-1}\left(\widetilde{u}_{\varphi}^{\mathrm{BGK}}+\frac{1}{8 \pi} \frac{\operatorname{Pr}-1}{\operatorname{Pr} k} \frac{h_{M}^{\mathrm{BGK}}}{r^{2}}\right), \\
& \widetilde{P}_{r \varphi}^{\mathrm{ES}}=\left(1+\frac{1}{8 \pi} \frac{\operatorname{Pr}-1}{\operatorname{Pr} k} h_{M}^{\mathrm{BGK}}\right)^{-1} \widetilde{P}_{r \varphi}^{\mathrm{BGK}}, \\
& \widetilde{Q}_{\varphi}^{\mathrm{ES}}=\left(1+\frac{1}{8 \pi} \frac{\operatorname{Pr}-1}{\operatorname{Pr} k} h_{M}^{\mathrm{BGK}}\right)^{-1} \widetilde{Q}_{\varphi}^{\mathrm{BGK}} .
\end{aligned}
$$

The moment of force acting on the sphere is also expressed as

$$
h_{M}^{\mathrm{ES}}=\left(1+\frac{1}{8 \pi} \frac{\operatorname{Pr}-1}{\operatorname{Pr} k} h_{M}^{\mathrm{BGK}}\right)^{-1} h_{M}^{\mathrm{BGK}} .
$$

With the aid of these relations, one can readily obtain the solution for the ES model from that for the BGK model $(\mathrm{Pr}=1$ or $\nu=0)$ for the common $k$ and $\alpha$. Moreover, the relation can be used to check the accuracy of numerical computation, if one has solutions for $\operatorname{Pr}=1$ and $\operatorname{Pr} \neq 1$ for the same $k$ and $\alpha$.

\section{Results for large and small $k$}

Before proceeding to the actual numerical analysis, we summarise here some analytical results available for large and small $k$. The formulas given in this section are not restricted to the ES model.

The solution in the case of a collisionless gas, i.e. $k=\infty$, is easily obtained for the present problem and is given by

$$
\phi_{S}= \begin{cases}2 \alpha r, & \left(0 \leqslant \theta_{\zeta}<\operatorname{Arcsin}\left(r^{-1}\right)\right) \\ 0, & \left(\operatorname{Arcsin}\left(r^{-1}\right)<\theta_{\zeta} \leqslant \pi\right) .\end{cases}
$$

Here, $\theta_{\zeta}\left(0 \leqslant \theta_{\zeta} \leqslant \pi\right)$ is the polar angle of the molecular velocity $\zeta_{i}$ measured from the radial direction, i.e. $\theta_{\zeta}=\operatorname{Arccos}\left(\zeta_{r} / \zeta\right)$. Thus, the solution is simply proportional to $\alpha$ in the case of the collisionless gas. The macroscopic quantities and the torque acting on the sphere are easily obtained and are summarised as follows:

$$
\begin{gathered}
\widetilde{u}_{\varphi}=\frac{\alpha r}{2}\left[1-\sqrt{1-\frac{1}{r^{2}}}\left(1+\frac{1}{2 r^{2}}\right)\right], \\
\widetilde{P}_{r \varphi}=\frac{\alpha}{\pi^{1 / 2}} \frac{1}{r^{3}}, \\
\widetilde{Q}_{\varphi}=0, \\
h_{M}=-\frac{8}{3} \pi^{1 / 2} \alpha .
\end{gathered}
$$




$\begin{array}{lccccc} & k_{0} & a_{1} & a_{2} & a_{3} & 3 k_{0}^{2}-3 a_{1}+a_{2}+a_{3} \\ \text { BGK } & -1.01619 & 0.76632 & 0.50000 & -0.26632 & 1.03265 \\ \text { ES (Pr =2/3) } & -0.67746 & 0.51088 & 0.33333 & -0.17755 & 0.00000 \\ \text { HS } & -1.25395 & 0.90393 & 0.66012 & -0.24381 & 2.42169\end{array}$

TABLE 1. The slip coefficients occurring in $(4.4 a)-(4.4 c)$ for the BGK model, the ES model with $\operatorname{Pr}=2 / 3$ and for the hard-sphere gas (HS) under the diffuse reflection boundary condition (or the Maxwell boundary condition with $\alpha=1$ ). Data taken from Sone (2007); Takata et al. $(2016 a)$.

Thus, the heat flow vanishes in the collisionless limit. From the above expression, it is easily seen that $\mathrm{d} \widetilde{u}_{\varphi} / \mathrm{d} r$ diverges with the rate $(r-1)^{-1 / 2}$ as $r \downarrow 1$.

The asymptotic expressions of the flow field for $k \ll 1$ are obtained with the aid of the asymptotic theory (the general slip flow theory) (Sone 2002, 2007). We summarise the results in the case of $\alpha=1$ :

$$
\begin{aligned}
\widetilde{u}_{\varphi} & =\frac{1}{r^{2}}+3 k\left(\frac{k_{0}}{r^{2}}+Y_{0}(\eta)\right)+3 k^{2}\left(\frac{3 k_{0}^{2}-3 a_{1}+a_{2}+a_{3}}{r^{2}}+\mathcal{Y}(\eta)\right)+\cdots, \\
\widetilde{P}_{r \varphi} & =\frac{3 \gamma_{1} k\left(1+3 k k_{0}+3 k^{2}\left(3 k_{0}^{2}-3 a_{1}+a_{2}+a_{3}\right)+\cdots\right)}{r^{3}}, \\
\widetilde{Q}_{\varphi} & =-3 k H_{A}(\eta)+\cdots,
\end{aligned}
$$

and

$$
h_{M}=-8 \pi \gamma_{1} k\left(1+3 k k_{0}+3 k^{2}\left(3 k_{0}^{2}-3 a_{1}+a_{2}+a_{3}\right)+\cdots\right),
$$

where

$$
\mathcal{Y}(\eta)=3 k_{0} Y_{0}(\eta)-3 Y_{a 1}(\eta)+Y_{a 2}(\eta)+Y_{a 3}(\eta),
$$

and $\eta=(r-1) / k$. We note that $\gamma_{1} k$ is the dimensionless viscosity $\left[(\sqrt{\pi} / 2) \gamma_{1} p_{\infty}\left(2 R T_{\infty}\right)^{-1 / 2} \ell_{\infty}\right.$ is the viscosity where $\gamma_{1}=1 /(1-\nu)=\operatorname{Pr}$ for the ES model, $\gamma_{1}=1$ for the BGK model and $\gamma_{1}=1.270042427$ for the hard-sphere model], $k_{0}, a_{1}, a_{2}$ and $a_{3}$ are the slip coefficients and that $Y_{0}(\eta), Y_{a 1}(\eta), Y_{a 2}(\eta), Y_{a 3}(\eta)$ and $H_{A}(\eta)$ are the Knudsen-layer functions (Sone 2007). The slip coefficients and the Knudsen-layer functions depend on the molecular model as well as on the model of the molecular scattering law on the surface. The values for the ES model under the diffuse reflection boundary condition, i.e. $\alpha=1$, have recently been obtained in Takata et al. (2016a). In this reference, it was shown that the above slip coefficients and the Knudsen-layer functions for the ES model are related to those for the BGK model (i.e. Pr $=1$ ) by the following simple relations:

$$
\begin{aligned}
& \left(k_{0}, a_{1}, a_{2}, a_{3}\right)_{\mathrm{ES}}=\operatorname{Pr}\left(k_{0}, a_{1}, a_{2}, a_{3}\right)_{\mathrm{BGK}}, \\
& \left(Y_{0}, Y_{a 1}, Y_{a 2}, Y_{a 3}, H_{A}\right)_{\mathrm{ES}}=\operatorname{Pr}\left(Y_{0}, Y_{a 1}, Y_{a 2}, Y_{a 3}, H_{A}\right)_{\mathrm{BGK}},
\end{aligned}
$$

where the subscripts "ES" and "BGK" stand for the the slip coefficients and Knudsenlayer functions for the ES model and those for the BGK model, respectively. We list the values of the slip coefficients for the BGK model and those for the ES model with $\operatorname{Pr}=2 / 3$ under the diffuse reflection boundary condition in table 1 . For the ES model with $\operatorname{Pr}=2 / 3$, the combination $3 k_{0}^{2}-3 a_{1}+a_{2}+a_{3}$, occurring at the third term in $\widetilde{u}_{\varphi}$, $\widetilde{P}_{r \varphi}$ and $h_{M}$, turns out to be practically zero.

We note that the S-layer corrections, which are required at the bottom of the Knudsen 
layer (Sone \& Takata 1992), have not been included in the above formulas for $\widetilde{u}_{\varphi}$ and $\widetilde{Q}_{\varphi}$ for $k \ll 1$.

\section{Numerical results}

We solved the boundary-value problem (2.15)-(2.17) numerically by a finite difference method. The main feature of the present problem is the propagation of the discontinuity of the velocity distribution function in the phase space along the characteristics $r \sin \theta_{\zeta}=$ $1\left[\theta_{\zeta}=\operatorname{Arccos}\left(\zeta_{r} / \zeta\right)\right]$. In order to capture this feature accurately, our method is based on a hybrid scheme consisting of a finite difference method and a method of characteristics. Its original form, among its variants, was developed for an evaporating flow from a cylindrical condensed phase (Sugimoto \& Sone 1992) and then applied to the problem of a slow uniform flow past a sphere (Takata et al. 1993). Recently, the process of calculating the discontinuity was refined in Taguchi \& Suzuki (2017). The numerical computations were carried out for $\operatorname{Pr}=1(\nu=0)$ and $\operatorname{Pr}=2 / 3(\nu=-0.5)$, and for various values of the accommodation coefficient $\alpha$.

It should be emphasised that the proper account of the discontinuity in the velocity distribution function is crucial for the purpose of the present study. Loyalka (1992) solved the same problem numerically by using the Legendre polynomial expansion (truncated at the fourth term) of the linearised collision kernel for a hard-sphere gas. However, probably due to his main interest on the global quantities (e.g. the torque), the discontinuity of the velocity distribution was not taken into account there. In the present study, numerical computations were carried out carefully and faithfully at the level of the velocity distribution function in order to achieve high accuracy required to reveal precise structures of the flow field. This is computationally quite challenging even with the ES or BGK model.

\subsection{Behaviour of the macroscopic quantities}

We first show the behaviour of the macroscopic quantities. Figure 1 shows the profiles of $u_{\varphi} / \Omega \sin \theta, P_{r \varphi} / \Omega \sin \theta$ and $Q_{\varphi} / \Omega \sin \theta$ in the case of the diffuse reflection $(\alpha=1)$ for $k=0.1,1$ and 10. The solid line indicates the results for $\operatorname{Pr}=2 / 3$ and dashed line those for $\operatorname{Pr}=1$ (or the BGK model). A flow is induced around the sphere due to the sphere rotation. The flow speed is faster for $\operatorname{Pr}=2 / 3$ than for $\operatorname{Pr}=1$ for the same $k$. On the other hand, the flow speed is larger for smaller $k$ and approaches the limit $u_{\varphi} \rightarrow \alpha \Omega \sin \theta / r^{2}$ as $k \rightarrow 0$. The tangential stress $P_{r \varphi}$ is inversely proportional to $r^{3}$ as seen from (2.24). There occurs a heat flux flowing in the opposite direction to the mass flow when $0<k<\infty$, in spite that the temperature is uniform. Note that this heat flow, however, vanishes in the collisionless limit (see $(4.2 c)$ ).

Next, in order to see the effect of the accommodation coefficient $\alpha$, the profiles of $u_{\varphi} / \Omega \sin \theta$ and $Q_{\varphi} / \Omega \sin \theta$ for various $\alpha(\alpha=1,0.6$ and 0.2$)$ are presented in figure 2 in the case of $\operatorname{Pr}=2 / 3(k=0.1,1$ and 10). The magnitude of the flow velocity and that of the heat flux decrease with the decrease of $\alpha$.

We have seen that the gradient of the tangential flow velocity $\partial u_{\varphi} / \partial r$ diverges on the boundary $r=1$ in the case of collisionless flow $(k=\infty)$. It is also seen from figures 1 and 2 that $u_{\varphi}$ and $Q_{\varphi}$ vary sharply near the boundary $r=1$. Though it is difficult to see from the figure, the heat flux $Q_{\varphi}$ for $\alpha=0.2$ is also seen to vary quite sharply near $r=1$ if the figure is enlarged. In order to see this behaviour more clearly, we show in figure 3 the variations of $u_{\varphi}$ and $Q_{\varphi}$ near the boundary as functions of $s=r-1$ for $k=10,1$ and 0.1 , in the case of the diffuse reflection boundary condition $(\alpha=1)$. Clearly, these quantity 


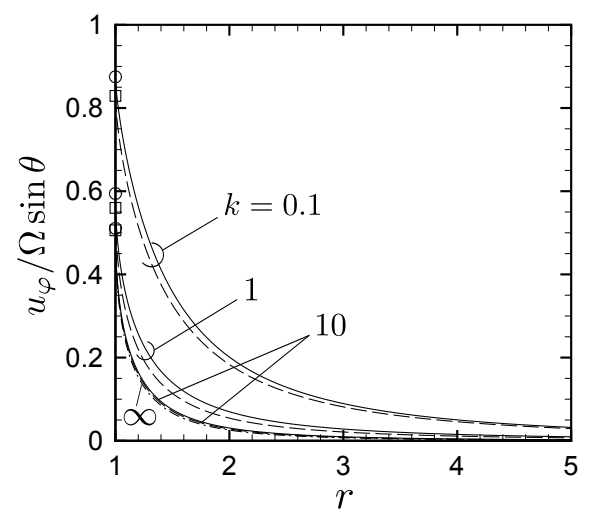

(a)

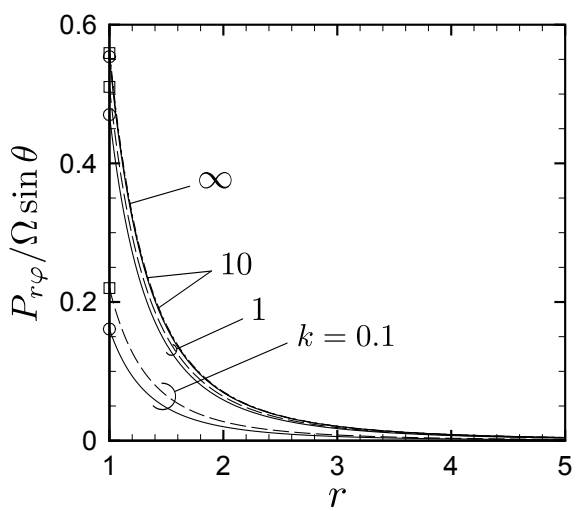

(b)

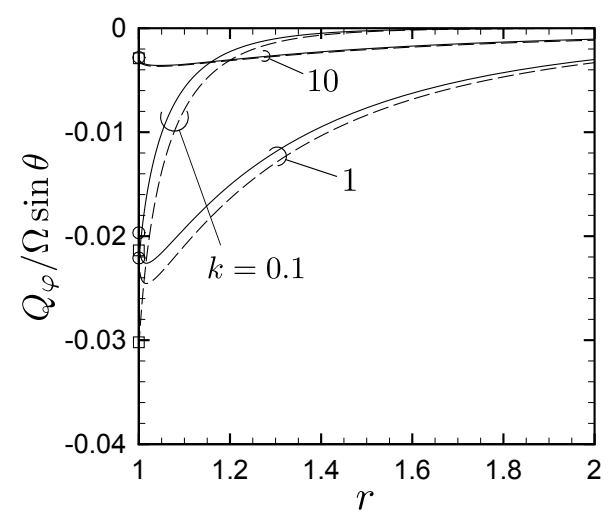

(c)

Figure 1. Profiles of the macroscopic quantities in the case of $\alpha=1$ (the diffuse reflection boundary condition). (a) $u_{\varphi}$, (b) $P_{r \varphi}$, (c) $Q_{\varphi}$. The solid line indicates the results for $\operatorname{Pr}=2 / 3$ and the dashed line those for $\operatorname{Pr}=1$. The value at $r=1$ is indicated by $\circ$ for $\operatorname{Pr}=2 / 3$ and by $\square$ for $\operatorname{Pr}=1$.

approach their boundary values in proportion to $s^{1 / 2}$ for each Knudsen number, implying that the divergence of $\partial u_{\varphi} / \partial r$ and $\partial Q_{\varphi} / \partial r$ occurs at $r=1$. It seems paradoxical from the conventional fluid mechanics view point, because the divergence of the derivative of the flow velocity implies that the viscous stress is not well-defined on the boundary. Note that however, the stress is not determined by the derivative of the flow velocity in a rarefied gas, but is directly related to the velocity distribution function. In figure 4, we show the variations of the same macroscopic variables for different values of $\alpha$ in the case of the Maxwell boundary condition for $k=10(\operatorname{Pr}=1)$. Again, we see the occurrence of the gradient divergence of the macroscopic variables on the boundary, implying that this phenomenon is not restricted to the case of the diffuse reflection boundary condition. In figure 4(b), several results based on different lattice systems (M1-3) are shown for $\alpha=0.2$ [(M1) is the finest and (M3) is the coarsest]. When the mesh near $r=1$ is refined, the variation tends to follow that of $s^{1 / 2}$. The cause of the gradient divergence is due to the propagation of the discontinuity of the velocity distribution function in the gas. We will come back to this point later in section 6 . For the moment, we continue to present our numerical results. 


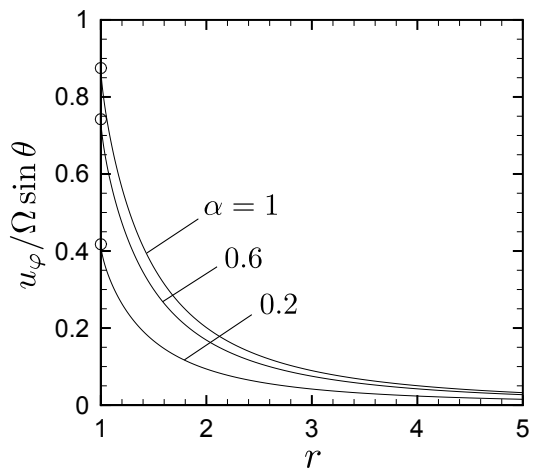

(a)

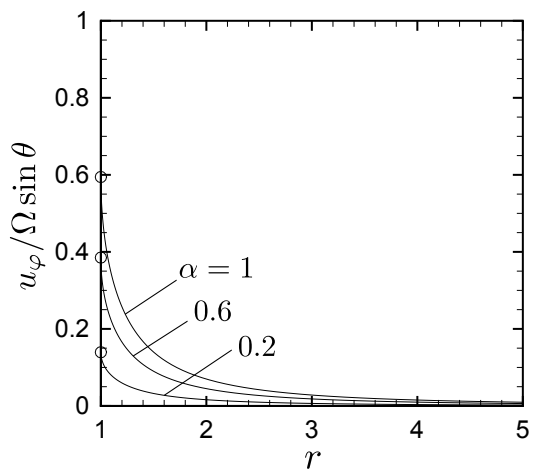

(c)

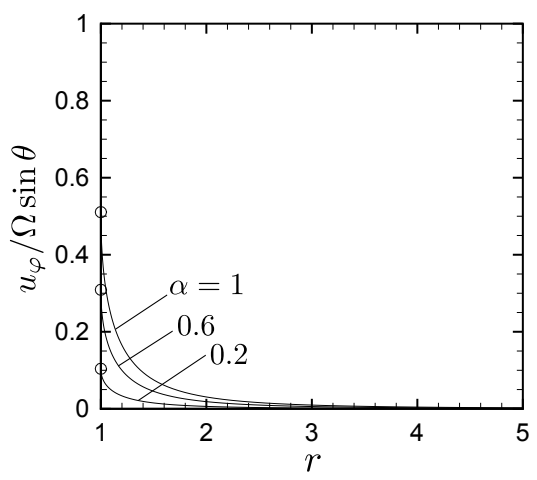

(e)

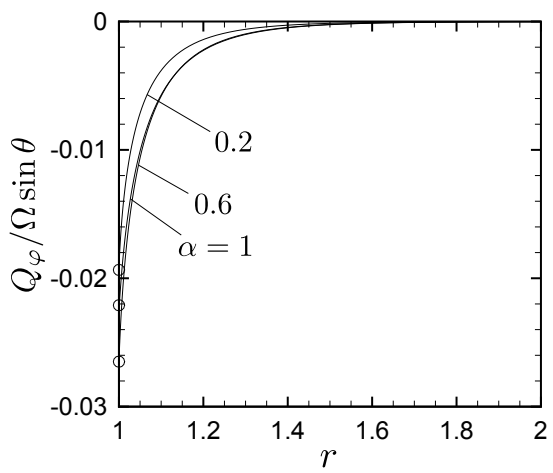

(b)

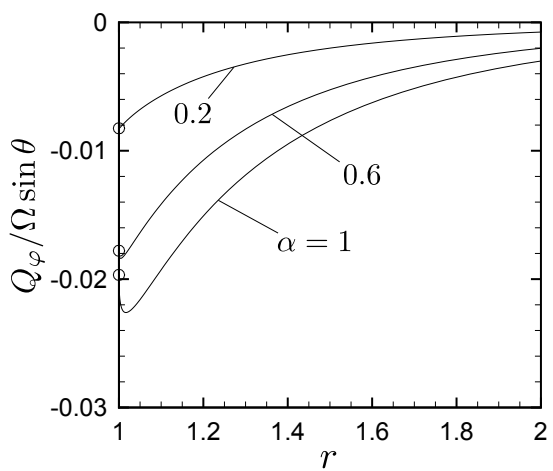

(d)

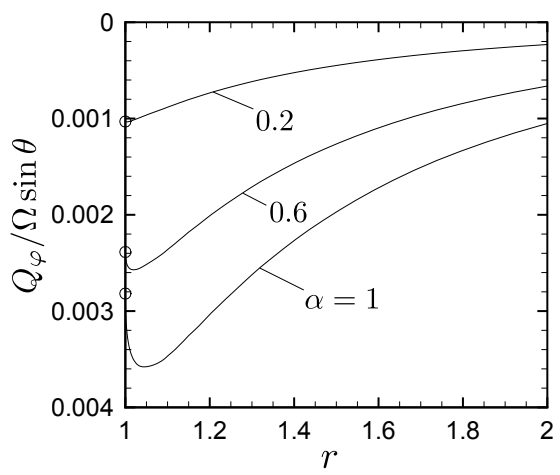

(f)

Figure 2. Profiles of $u_{\varphi}$ and $Q_{\varphi}$ for various $\alpha$ in the case of $\operatorname{Pr}=2 / 3$. (a,b) $k=0.1$, (c,d) $k=1,(\mathrm{e}, \mathrm{f}) k=10$. The value at $r=1$ is indicated by $\circ$.

\subsection{Moment of force acting on the sphere}

We now show the results for the (dimensionless) moment of force $h_{M}$ acting on the sphere. Figure 5 shows $h_{M}$ as a function of $k$ for $\operatorname{Pr}=1$ and $2 / 3$ and for various values of $\alpha(\alpha=1,0.6$ and 0.2$)$. The symbols represent the results of the direct numerical analysis. For comparison, the values of $h_{M}$ for $\operatorname{Pr}=2 / 3$ are also calculated from those of $\operatorname{Pr}=1$ with the aid of the formula (3.8) and are shown by the symbol + in figure $5(\mathrm{~b})$. 


$\begin{array}{cccccc}k & \alpha=0.2 & \alpha=0.4 & \alpha=0.6 & \alpha=0.8 & \alpha=1 \\ 0.1 & 0.7179 & 1.1589 & 1.4588 & 1.6771 & 1.8439 \\ 0.2 & 0.8283 & 1.4753 & 1.9960 & 2.4253 & 2.7861 \\ 0.3 & 0.8695 & 1.6106 & 2.2510 & 2.8106 & 3.3047 \\ 0.4 & 0.8902 & 1.6829 & 2.3940 & 3.0362 & 3.6197 \\ 0.5 & 0.9024 & 1.7269 & 2.4837 & 3.1814 & 3.8271 \\ 0.6 & 0.9104 & 1.7562 & 2.5445 & 3.2814 & 3.9723 \\ 0.7 & 0.9159 & 1.7769 & 2.5881 & 3.3541 & 4.0789 \\ 0.8 & 0.9200 & 1.7923 & 2.6208 & 3.4090 & 4.1601 \\ 0.9 & 0.9231 & 1.8042 & 2.6462 & 3.4519 & 4.2239 \\ 1 & 0.9256 & 1.8136 & 2.6663 & 3.4861 & 4.2751 \\ 2 & 0.9361 & 1.8541 & 2.7547 & 3.6384 & 4.5058 \\ 3 & 0.9393 & 1.8669 & 2.7829 & 3.6876 & 4.5813 \\ 4 & 0.9409 & 1.8730 & 2.7966 & 3.7117 & 4.6185 \\ 5 & 0.9418 & 1.8767 & 2.8047 & 3.7260 & 4.6405 \\ 6 & 0.9424 & 1.8791 & 2.8100 & 3.7354 & 4.6551 \\ 7 & 0.9428 & 1.8808 & 2.8138 & 3.7421 & 4.6655 \\ 8 & 0.9432 & 1.8820 & 2.8166 & 3.7470 & 4.6733 \\ 9 & 0.9434 & 1.8830 & 2.8188 & 3.7509 & 4.6793 \\ 10 & 0.9436 & 1.8838 & 2.8206 & 3.7540 & 4.6840\end{array}$

TABLE 2. Values of $-h_{M}$ for various $k$ and $\alpha$ on the basis of the BGK model (or the ES model with $\operatorname{Pr}=1$ ) under the Maxwell boundary condition with accommodation coefficient $\alpha$.

$\begin{array}{cccccccccc}k & \alpha=0.2 & & \alpha=0.4 & \alpha=0.6 & & \alpha=0.8 & & \alpha=1 & \\ 0.1 & 0.6282 & (0.6282) & 0.9417 & 1.1306 & - & 1.2575 & (1.2575) & 1.3490 & (1.3490) \\ 0.2 & 0.7653 & - & 1.2865 & 1.6654 & - & 1.9539 & (1.9538) & 2.1815 & (2.1814) \\ 0.3 & 0.8221 & - & 1.4552 & 1.9586 & (1.9586) & 2.3691 & (2.3690) & 2.7106 & (2.7105) \\ 0.4 & 0.8524 & - & 1.5529 & 2.1393 & (2.1392) & 2.6379 & (2.6378) & 3.0674 & (3.0674) \\ 0.5 & 0.8711 & - & 1.6158 & 2.2603 & - & 2.8239 & - & 3.3213 & (3.3212) \\ 0.6 & 0.8837 & - & 1.6595 & 2.3465 & - & 2.9594 & - & 3.5100 & (3.5099) \\ 0.7 & 0.8927 & - & 1.6915 & 2.4108 & - & 3.0622 & - & 3.6552 & (3.6551) \\ 0.8 & 0.8994 & - & 1.7158 & 2.4605 & - & 3.1426 & - & 3.7701 & (3.7700) \\ 0.9 & 0.9047 & - & 1.7350 & 2.4999 & - & 3.2072 & - & 3.8632 & (3.8631) \\ 1 & 0.9089 & (0.9089) & 1.7504 & 2.5320 & - & 3.2600 & - & 3.9400 & (3.9400) \\ 2 & 0.9275 & - & 1.8206 & 2.6813 & - & 3.5113 & - & 4.3125 & (4.3125) \\ 3 & 0.9335 & - & 1.8440 & 2.7325 & - & 3.5996 & - & 4.4462 & (4.4462) \\ 4 & 0.9365 & - & 1.8558 & 2.7582 & - & 3.6444 & - & 4.5147 & (4.5147) \\ 5 & 0.9383 & - & 1.8628 & 2.7737 & - & 3.6715 & - & 4.5564 & (4.5564) \\ 6 & 0.9395 & - & 1.8674 & 2.7841 & - & 3.6897 & - & 4.5844 & (4.5844) \\ 7 & 0.9403 & - & 1.8708 & 2.7915 & - & 3.7027 & - & 4.6045 & (4.6045) \\ 8 & 0.9409 & - & 1.8733 & 2.7970 & - & 3.7124 & - & 4.6196 & (4.6196) \\ 9 & 0.9414 & - & 1.8752 & 2.8014 & - & 3.7201 & - & 4.6314 & (4.6313) \\ 10 & 0.9418 & (0.9418) & 1.8767 & 2.8048 & - & 3.7261 & - & 4.6408 & (4.6408)\end{array}$

TABLE 3. Values of $-h_{M}$ for various $k$ and $\alpha$ on the basis of the ES model with $\operatorname{Pr}=2 / 3$ under the Maxwell boundary condition with accommodation coefficient $\alpha$. The results were obtained from those for $\mathrm{Pr}=1$ with the aid of the formula (3.8). The results of direct numerical computations for $\operatorname{Pr}=2 / 3$ are shown in the parentheses. 


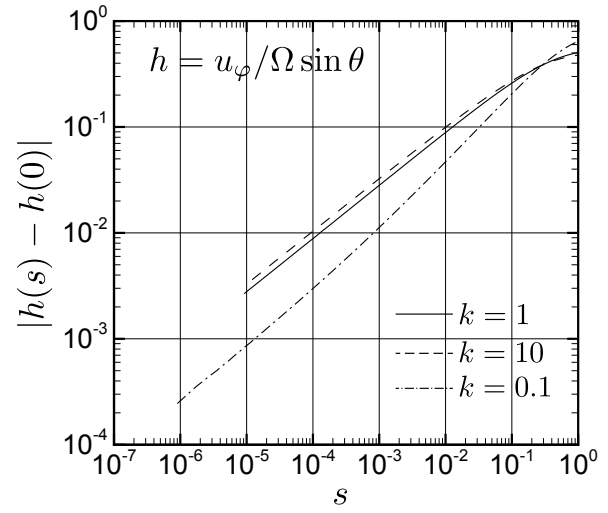

(a)

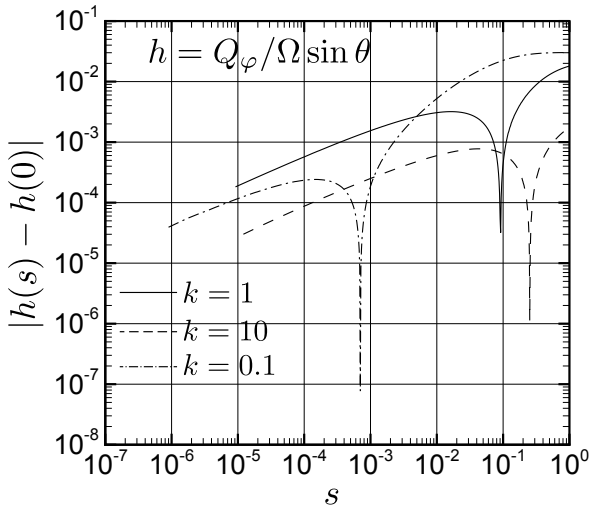

(b)

FIGURE 3. Variations of $u_{\varphi}$ and $Q_{\varphi}$ near the surface of the sphere as functions of the normal distance $s=r-1$ for various $k(\operatorname{Pr}=1, \alpha=1)$. (a) $u_{\varphi}$, (b) $Q_{\varphi}$.

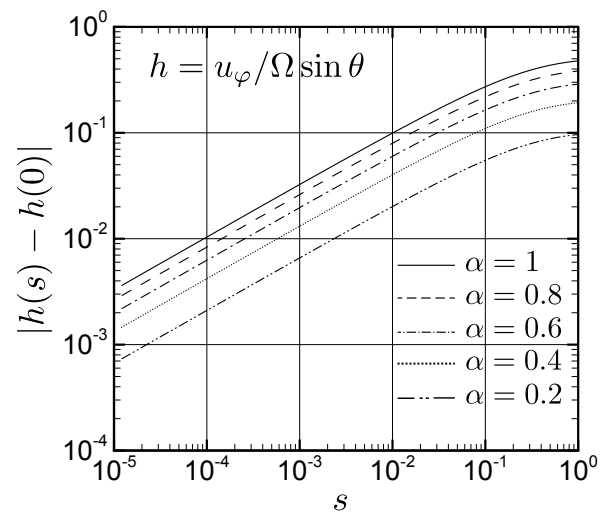

(a)

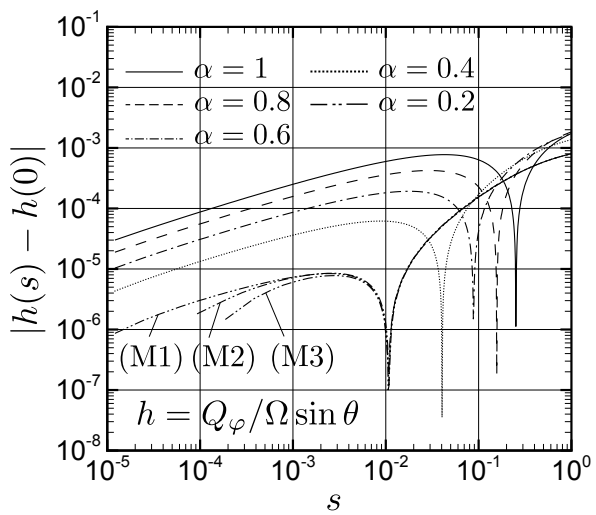

(b)

FIgURE 4. Variations of $u_{\varphi}$ and $Q_{\varphi}$ near the surface of the sphere as functions of the normal distance $s=r-1$ for various $\alpha(\operatorname{Pr}=1, k=10)$. (a) $u_{\varphi}$, (b) $Q_{\varphi}$. (M1-3) in the panel (b) are the results based on different lattice systems; (M1) is the finest and (M3) is the coarsest.

The results of the direct numerical computations and those obtained from the formula agree well (see also table 3 ). The corresponding values for $\operatorname{Pr}=1$ and those for $\operatorname{Pr}=2 / 3$ are tabulated in tables 2 and 3, respectively, where the results for $\alpha=0.8$ and 0.4 are also included. The magnitude of $h_{M}$ increases monotonically with $k$, and approaches the limiting value $h_{M} \rightarrow-(8 / 3) \pi^{1 / 2} \alpha$ as $k \rightarrow \infty$. The moment of force decreases in its magnitude with the decrease of the accommodation coefficient $\alpha$. The two-terms asymptotic formula and three-terms formula for $\alpha=1$ (the dash-dotted line and the solid line) do not make difference in the case of $\operatorname{Pr}=2 / 3$, since the coefficient of the term $k^{3}$ is zero within the significant figures (see table 1). Incidentally, the asymptotic formula for the Maxwell boundary condition with $\alpha \in(0,1)$ requires the information on the slip coefficients $\left(k_{0}, a_{1}, a_{2}, a_{3}\right)$ for $\alpha \neq 1$. The values of the first-order slip coefficient $k_{0}$ under the Maxwell boundary condition were obtained in Wakabayashi et al. (1996) for various $\alpha$, for the hard-sphere gas. Results based on the variational approach are 


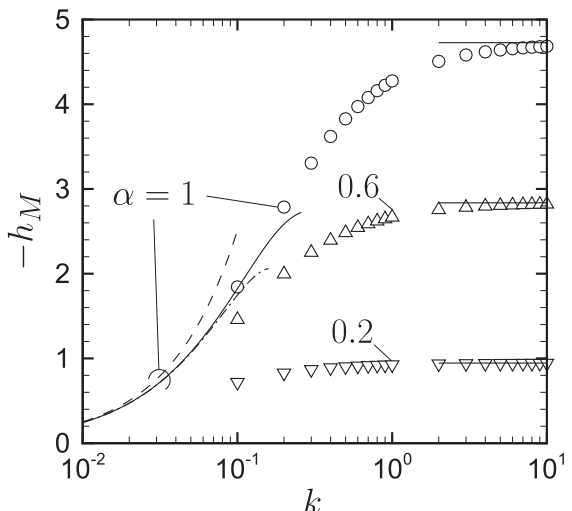

(a)

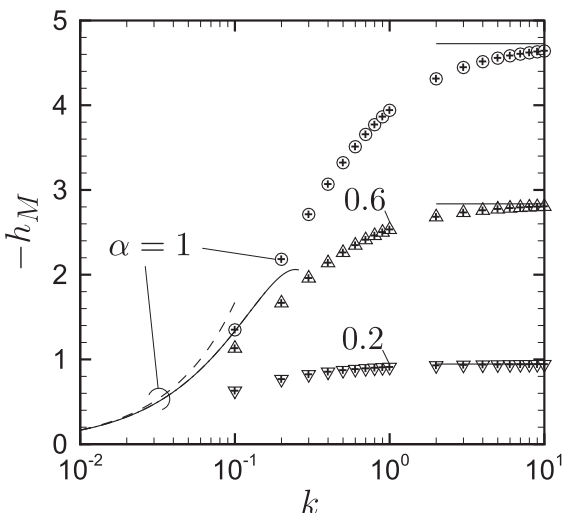

(b)

Figure $5 . h_{M}$ versus $k$ on the basis of the ES model under the Maxwell boundary condition with accommodation coefficient $\alpha$. (a) $\operatorname{Pr}=1$ (or the BGK model), (b) $\operatorname{Pr}=2 / 3$. The symbol o indicates the numerical results. The horizontal lines indicate the values in the collisionless gas limit $(k \rightarrow \infty)$. The results based on the asymptotic formula (4.5) for $\alpha=1$ are shown by the broken line (one term), the dash-dotted line (two terms) and by the solid line (three terms). In (b), the symbol + represents the result obtained from that for $\operatorname{Pr}=1$ with the aid of formula (3.8).

available in Loyalka \& Hickey (1989). The leading-order term of the formula is given by $h_{M}=-8 \pi \gamma_{1} \alpha k$.

The increasing trend of $-h_{M}$ in terms of $k$ can be interpreted in the case of large and small $k$ as follows. For the sake of convenience of discussion, we take the frame of reference rotating with the sphere, in which the sphere is at rest and the fluid is rotating. Also for clarity, we consider the situation where the molecules are reflected diffusely on the surface. In this case, the reflected molecules have an isotropic velocity distribution and give no contribution to the tangential momentum flux on the surface at a point under consideration. Then, the torque acting on the sphere is determined by solely the tangential momentum flux carried by the impinging molecules on the boundary. For the free molecular flow, all the impinging molecules come directly from the infinity. When $k$ is large but finite, some molecules, after having reflected on the surface, collide with the incoming molecules and hit them back, thereby reducing the momentum flux transmitted to the boundary. The torque is therefore reduced with the decrease of $k$ when $k$ is large. On the other hand, when $k$ is small, the molecules coming from the region several mean free paths away from the surface essentially determine the momentum flux. Since there is a shear around the sphere, the molecules arriving at a point on the surface have faster tangential velocity when $k$ bocomes larger. Therefore, the torque is increasing with $k$, when $k$ is small.

Finally, compare $h_{M}$ for different $\operatorname{Pr}$ in the case of $\alpha=1$ (i.e. the diffuse reflection boundary condition) in figure 6 . Here, the results for $\operatorname{Pr}>1$ are also included though it is unrealistic for a gas. $-h_{M}$ increases with the increase of $\mathrm{Pr}$. However, if $\mathrm{Pr}$ is further increased, the monotonicity of $-h_{M}$ with respect to $k$ is lost.

REMARK 1. We have so far confined our consideration to the case of a monatomic gas. The extension to the case of a polyatomic gas is simpe if we adopt the ES model for a polyatomic gas proposed by Andries et al. (2000) to replace our basic equation (with a suitable modification in the diffuse reflection boundary condition). Let us denote by $\delta$ the number of degrees of freedom of a gas molecule, by $R T_{\infty} \epsilon$ the energy related to the 


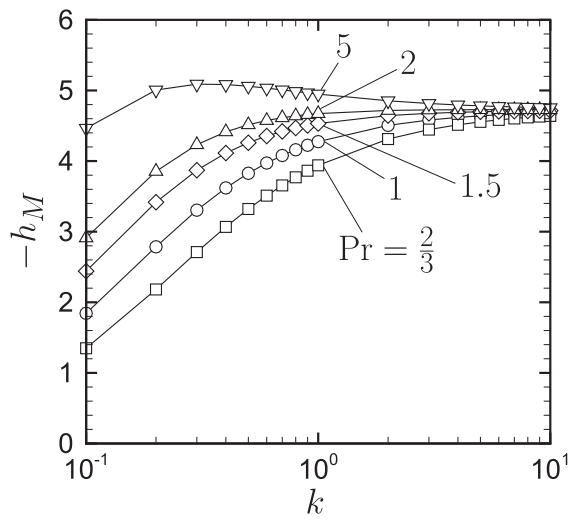

Figure $6 . h_{M}$ vs $k$ for various $\operatorname{Pr}$ in the case of $\alpha=1$ (the diffuse reflection condition). The symbols show the numerical results based on the ES model, which are connected by the solid lines.

internal degree of freedom and by $\rho_{\infty}\left(2 R T_{\infty}\right)^{-3 / 2}\left(R T_{\infty}\right)^{-1}(1+\bar{\phi}(\boldsymbol{x}, \boldsymbol{\zeta}, \epsilon)) E\left(\zeta_{i}\right) E_{\delta}(\epsilon)$ the molecular velocity distribution function, where $E_{\delta}(\epsilon)=\Lambda_{\delta} \epsilon^{\delta / 2-1} \exp (-\epsilon)$ with $\Lambda_{\delta}^{-1}=$ $\int_{0}^{\infty} \epsilon^{\delta / 2-1} \exp (-\epsilon) \mathrm{d} \epsilon$. We also introduce the following notations for the polyatomic gas under consideration: $\ell_{\infty}^{*}=(2 / \sqrt{\pi})\left(2 R T_{\infty}\right)^{1 / 2} / A_{c}^{*} \rho_{\infty}$ with $A_{c}^{*}$ being a constant is the molecular mean free path at the reference equilibrium state at rest, $K n^{*}=\ell_{\infty}^{*} / L, k^{*}=$ $(\sqrt{\pi} / 2) K n^{*}, \alpha^{*} \in[0,1]$ the accommodation coefficient $\dagger$ and

$$
\operatorname{Pr}^{*}=\frac{\delta+5}{2} \frac{R \mu_{\infty}^{*}}{\lambda_{\infty}^{*}}
$$

the Prandtl number, where $\mu_{\infty}^{*}$ and $\lambda_{\infty}^{*}$ are, respectively, the viscosity and the thermal conductivity at the reference state. Then, if we introduce the similarity solution similar to (2.14) as well as its the marginal with respect to the energy related to the internal degree of freedom, i.e.

$$
\bar{\phi}=\Omega \zeta_{\varphi} \bar{\phi}_{S}\left(r, \zeta_{r}, \zeta, \epsilon\right) \sin \theta \quad \text { and } \quad \mathcal{F}_{S}\left(r, \zeta_{r}, \zeta\right)=\int_{0}^{\infty} \bar{\phi}_{S} E_{\delta} \mathrm{d} \epsilon,
$$

it turns out that $\mathcal{F}_{S}$ solves the same equation and boundary conditions as $\phi_{S},(2.15)-$ (2.19), provided that the following correspondence between the parameters is satisfied:

$$
k^{*}=k, \quad \alpha^{*}=\alpha, \quad \operatorname{Pr}^{*}=\operatorname{Pr}=1 /(1-\nu) .
$$

Also under the same condition, the macroscopic variables of the polyatomic gas also coincide with those of a monatomic gas. Therefore, the present result for a monatomic gas also gives the result for the case of a polyatomic gas. This also signifies the utility of the conversion formula derived in section 3.

We conclude this section by a brief summary of the present numerical analysis.

- The flow speed is faster for $\operatorname{Pr}=2 / 3$ than for $\operatorname{Pr}=1$ and faster for smaller $k$.

- There exists nonzero heat flux in the gas in spite that the temperature of the gas is uniform. The heat flow vanishes at the two limits $k=\infty$ and 0 .

- The flow and heat flow decrease as the accommodation coefficient $(\alpha)$ becomes small.

- The tangential component of the flow velocity and that of the heat flux, $u_{\varphi}$ and

$\dagger$ For the present linearised problem, the Maxwell boundary condition on the sphere is given $\stackrel{\text { by }}{\bar{\phi}}=\left(1-\alpha^{*}\right) \bar{\phi}\left(x_{i}, \zeta_{i}-2 \zeta_{r} n_{i}, \epsilon\right)+\alpha^{*}\left(-2 \sqrt{\pi} \int_{\zeta_{r}<0} \int_{0}^{\infty} \zeta_{r} \bar{\phi} E E_{\delta} \mathrm{d} \epsilon \mathrm{d} \zeta+2 \Omega \zeta_{\varphi} \sin \theta\right), \quad \zeta_{r}>0, \quad(r=1)$. 
$Q_{\varphi}$, vary abruptly near the boundary in a way that their normal derivatives diverge on the boundary with the rate $(r-1)^{-1 / 2}$. On the other hand, as $(2.24)$ implies, such a divergence of the normal derivative does not occur for $P_{r \varphi}$. Such observations are not peculiar to the diffuse reflection boundary condition.

- $-h_{M}(\geqslant 0)$ is monotonically increasing in $k$ if Pr is not very large.

- The present result is also applicable to the case of a polyatomic gas.

\section{Discussions: gradient divergence}

We have seen that the normal derivatives of the macroscopic quantities $u_{\varphi}$ and $Q_{\varphi}$ diverge on the boundary. The present section discusses the cause of the occurrence of gradient divergence in more detail along the line of Takata \& Taguchi (2017). The point is the propagation of the discontinuity of the velocity distribution function along the characteristics $r \sin \theta_{\zeta}=1$ in the phase space.

Let us consider the tangential flow velocity $u_{\varphi}$ as an example, whose radial dependency $\widetilde{u}_{\varphi}(r)\left(=u_{\varphi} / \Omega \sin \theta\right)$ is given by

$$
\tilde{u}_{\varphi}=\pi \int_{0}^{\infty} \int_{0}^{\pi} \zeta^{4} \sin ^{3} \theta_{\zeta} \phi_{S}\left(r, \theta_{\zeta}, \zeta\right) E \mathrm{~d} \theta_{\zeta} \mathrm{d} \zeta .
$$

Here, $\phi_{S}$ is regarded as a function of $\left(r, \theta_{\zeta}, \zeta\right)$. Now, keeping in mind that $\phi_{S}$ is discontinuous at $\theta_{\zeta}=\theta_{\zeta}^{*} \equiv \operatorname{Arcsin}\left(r^{-1}\right)$, we differentiate the above expression with respect to $r$ to obtain

$$
\frac{\mathrm{d} \widetilde{u}_{\varphi}}{\mathrm{d} r}=\pi \int_{0}^{\infty} \int_{0}^{\pi} \zeta^{4} \sin ^{3} \theta_{\zeta} \frac{\partial \phi_{S}}{\partial r} E \mathrm{~d} \theta_{\zeta} \mathrm{d} \zeta-\pi \int_{0}^{\infty} \zeta^{4}\left[\phi_{S}\right]^{ \pm} \sin ^{3} \theta_{\zeta}^{*} \frac{\mathrm{d} \theta_{\zeta}^{*}}{\mathrm{~d} r} E \mathrm{~d} \zeta
$$

where

$$
\left[\phi_{S}\right]^{ \pm}=\phi_{S}\left(r, \theta_{\zeta}^{*}+0, \zeta\right)-\phi_{S}\left(r, \theta_{\zeta}^{*}-0, \zeta\right)
$$

The second term arises because the location of the discontinuity $\theta_{\zeta}=\theta_{\zeta}^{*}(r)$ changes with $r$. Now substituting the explicit form of $\theta_{\zeta}^{*}$, the second term is further transformed into

$$
\text { (second term) }=\frac{\pi}{r^{4} \sqrt{r^{2}-1}} \int_{0}^{\infty} \zeta^{4}\left[\phi_{S}\right]^{ \pm} E \mathrm{~d} \zeta,
$$

which diverges with the rate $(r-1)^{-1 / 2}$ as $r \downarrow 1$, provided that $\left|\int_{0}^{\infty} \zeta^{4}\left[\phi_{S}\right]^{ \pm} E \mathrm{~d} \zeta\right|$ is bounded from below by a positive constant. On the other hand, the first term diverges at most logarithmically on approaching the boundary. We leave the estimate of the first term in Appendix B. Thus, the normal derivative $\partial u_{\varphi} / \partial r$ diverges on the boundary with the diverging rate $(r-1)^{-1 / 2}$ due to the second term of (6.2). Since its mechanism is the propagation of the discontinuity of VDF into the gas, it should be observed irrespective of the magnitude of $k$ (even in the free molecular gas limit). Similarly, we can show that $\partial Q_{\varphi} / \partial r$ diverges with the same rate, $(r-1)^{-1 / 2}$, as $r \downarrow 1$, which is also consistent with our numerical results.

Now let us introduce the following notation for the integral measuring the magnitude of jump across $r \sin \theta_{\zeta}=1$ (a weighted marginal with respect to the $\zeta$-variable):

$$
G(r)=\int_{0}^{\infty} \zeta^{4}\left[\phi_{S}\right]^{ \pm} E \mathrm{~d} \zeta
$$

From the above discussion, $\widetilde{u}_{\varphi}$ can be expressed as $\widetilde{u}_{\varphi}=\left.\widetilde{u}_{\varphi}\right|_{r=1}+C_{1}(r-1)^{1 / 2}+\cdots$ for $r \sim 1$, and the coefficient $C_{1}$ of the leading term of the diverging gradient should be 


\begin{tabular}{cccccccc}
$k=10$ & & \multicolumn{7}{c}{$k=1$} & & \\
$\alpha$ & $C_{1}$ & $G_{0}$ & $C_{1} / G_{0}$ & $\alpha$ & $C_{1}$ & $G_{0}$ & $C_{1} / G_{0}$ \\
1 & -1.0380 & -0.2343 & 4.431 & 1 & -0.8796 & -0.1976 & 4.453 \\
0.8 & -0.8334 & -0.1881 & 4.430 & 0.8 & -0.7301 & -0.1641 & 4.450 \\
0.6 & -0.6274 & -0.1416 & 4.430 & 0.6 & -0.5683 & -0.1277 & 4.449 \\
0.4 & -0.4198 & -0.0948 & 4.429 & 0.4 & -0.3933 & -0.0884 & 4.447 \\
0.2 & -0.2106 & -0.0476 & 4.428 & 0.2 & -0.2042 & -0.0459 & 4.445
\end{tabular}

TABLE 4 . Values of $C_{1}$ and $G_{0}=\lim _{r \downarrow 1} \int_{0}^{\infty} \zeta^{4}\left[\phi_{S}\right]^{ \pm} E \mathrm{~d} \zeta$ for various accommodation coefficients $\alpha(k=10,1)$.

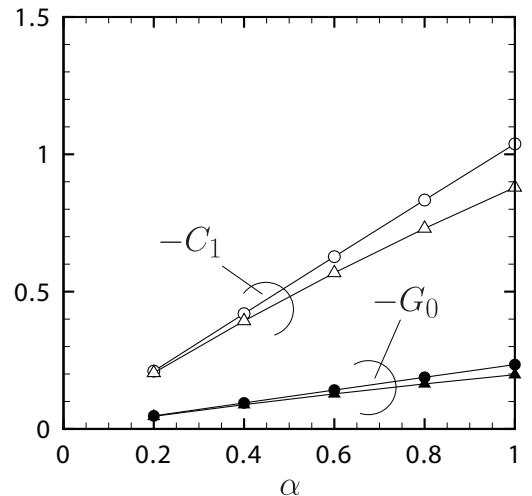

(a)

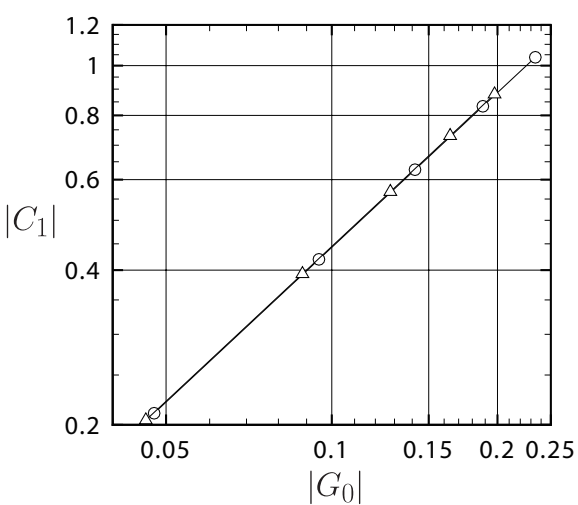

(b)

FiguRE 7. (a) $C_{1}$ and $G_{0}=\lim _{r \downarrow 1} \int_{0}^{\infty} \zeta^{4}\left[\phi_{S}\right]^{ \pm} E \mathrm{~d} \zeta$ for various accommodation coefficients $\alpha$ in the cases of $k=10$ and $1(\operatorname{Pr}=1)$. The circles $(\circ, \bullet)$ are for the case $k=10$ and the triangles $(\triangle, \mathbf{\Delta})$ for the case $k=1$. (b) Double-log plot of $\left|C_{1}\right|$ vs $\left|G_{0}\right|$ for $k=10$ (o) and $1(\triangle)$. In both figures, the symbols represent the numerical results which are connected by the solid lines.

related to $G_{0} \equiv \lim _{r \rightarrow 1+0} G(r)$, as

$$
C_{1}=2^{1 / 2} \pi G_{0}
$$

where the factor $2^{1 / 2} \pi$ is purely geometric. To check this relation, the value of $C_{1}$ was obtained by fitting the curve $\widetilde{u}_{\varphi}=\left.\widetilde{u}_{\varphi}\right|_{r=1}+C_{1}(r-1)^{1 / 2}$ to the numerical data by the least square method using five data points adjacent to the boundary. At the same time, the value of $G_{0}(=G(1))$ was calculated numerically using the data of $\phi_{S}$ at $\theta_{\zeta}=\pi / 2 \pm 0$ on the boundary. We show the values of $C_{1}$ and $G_{0}$ thus obtained in table 4 and in figure 7 for $k=10$ and 1 and for various $\alpha$ in the case of $\operatorname{Pr}=1$. It is clearly observed that $C_{1}$ varies in proportion to $G_{0}$, a measure of the magnitude of jump discontinuity of VDF (see figure $7(\mathrm{~b})$ ). The constant of proportionality is, according to (6.6), $2^{1 / 2} \pi \sim 4.44288$ irrespective of $\alpha>0$. As is seen from the columns $C_{1} / G_{0}$ in table 4 , our numerical results show $C_{1} / G_{0} \sim 4.4$, which is close to $2^{1 / 2} \pi$, and again support the discussion in this section.

As for $P_{r \varphi}$, a factor $\zeta_{r}=\zeta \cos \theta_{\zeta}$ is contained in the integrand. This factor acts to cancel the singularity originated from $\mathrm{d} \theta_{\zeta}^{*} / \mathrm{d} r$. This explains why $\partial P_{r \varphi} / \partial r$ remains finite as $r \downarrow 1$. This result is also consistent with the more general result that the normal derivative of any moment which contains $\zeta_{i} n_{i}$ as a factor in the integrand $\left(n_{i}\right.$ is the unit normal vector to the boundary) do not diverge on a smooth boundary (Takata \& Taguchi 2017). 
We conclude this section with a brief comment on the S layer. The discontinuity of the velocity distribution function decays appreciably over the distance of the order of the mean free path due to the molecular collision. Therefore, when $k$ is small, the region where the discontinuity of $\mathrm{VDF}$ is appreciable is confined in a thin region adjacent to the boundary with the thickness of order $L k^{2}$. This thin region at the bottom of the Knudsen layer whose thickness is of order $L k$, is the S layer (Sone 1973; Sone \& Takata 1992). The discussion in the present section is applicable irrespective of the values of $k$, and therefore naturally applies to the $\mathrm{S}$ layer. In this way, the pesent result also clarify the structure of the $\mathrm{S}$ layer around a rotating sphere.

\section{Conclusion}

In this paper, we have studied a flow induced around a spinning sphere in a rarefied gas in detail, on the basis of the linearised ES model and Maxwell diffuse-specular boundary condition. The main results are summarised as follows:

- We have derived a conversion formula that allows us to derive the result for arbitrary $\operatorname{Pr}(\geqslant 2 / 3)$ from that for $\operatorname{Pr}=1$, for given $(k, \alpha)$.

- We have clarified the detailed profiles of the macroscopic quantities (flow velocity, tangential stress, heat flow). In particular, we have shown numerically that the normal derivatives of $u_{\varphi}$ and $Q_{\varphi}$ diverge on the boundary with the rate $1 / \sqrt{r-1}$, which is consistent with the estimate obtained in Takata \& Taguchi (2017). The diverging term in the normal derivatives of $u_{\varphi}$ and $Q_{\varphi}$ are proportional to the magnitude of the jump discontinuity in VDF on the boundary.

- On the other hand, the normal derivative of $P_{r \varphi}$ does not diverge on approaching the boundary. The result is consistent with the more general result that the normal derivative of any moment containing $\zeta_{i} n_{i}$ as a factor in the integrand $\left(n_{i}\right.$ : unit normal vector to the boundary) do not diverge on a smooth boundary.

- We have obtained the moment of force acting on the sphere for a wide range of the parameter space.

- The present result is also applicable to the case of a polyatomic gas.

This work was supported by JSPS KAKENHI Grant Number 25820041 and 17H03173 and in part by JSPS KAKENHI Grant Number 17K06146. The authors also acknowledge the support by JSPS and MAEDI under the Japan-France Integrated Action Program (SAKURA).

\section{Appendix A. On the temperature of the sphere}

In this paper, we have assumed that the temperature of the sphere is uniform and is equal to that of the gas at infinity. In this appendix, we justify this in the case where the heat flow in the sphere is described by the Fourier law.

We take the linearised Boltzmann equation as our basic equation and assume the general kinetic boundary condition on the sphere, which includes the ES model and the Maxwell boundary condition as particular examples. Let $\rho_{\infty}\left(2 R T_{\infty}\right)^{-3 / 2}(1+\phi(\boldsymbol{x}, \boldsymbol{\zeta})) E$ be the velocity distribution function of the gas molecules, $T_{\infty}\left(1+\tau_{s}(\boldsymbol{x})\right)$ the temperature of the sphere, $\left(2 R T_{\infty}\right)^{-3 / 2} K_{B 0}\left(\boldsymbol{\zeta}, \boldsymbol{\zeta}^{*}\right)$ the scattering kernel at the reference equilibrium state at rest describing the relation between the velocities of the incident molecules $\boldsymbol{\zeta}^{*}\left(\zeta_{r}^{*}<0\right)$ and those of the reflected molecules $\boldsymbol{\zeta}\left(\zeta_{r}>0\right)$ on the surface and $p_{\infty}\left(2 R T_{\infty}\right)^{1 / 2}\left(L / T_{\infty}\right) \hat{\lambda}_{s}(>0)$ the thermal conductivity of the sphere. The other notations appearing below are the same as those in the main text, unless otherwise stated. Then, 
$\phi$ and $\tau_{s}$ satisfy the following equations and boundary conditions:

$$
\begin{gathered}
\zeta_{i} \frac{\partial \phi}{\partial x_{i}}=\frac{1}{k} \mathcal{L}[\phi], \quad(|\boldsymbol{x}|>1), \\
\phi=g_{w}+E^{-1} \int_{\zeta_{r}^{*}<0} K_{B 0}\left(\boldsymbol{\zeta}, \boldsymbol{\zeta}^{*}\right)\left(\phi^{*}-g_{w}^{*}\right) E^{*} \mathrm{~d} \boldsymbol{\zeta}^{*}, \quad \zeta_{r}>0, \quad(|\boldsymbol{x}|=1), \\
\phi \rightarrow 0, \quad(|\boldsymbol{x}| \rightarrow \infty), \\
\frac{\partial}{\partial x_{j}}\left(\hat{\lambda}_{s} \frac{\partial \tau_{s}}{\partial x_{j}}\right)=0, \quad(|\boldsymbol{x}|<1), \\
\hat{\lambda}_{s} \frac{\partial \tau_{s}}{\partial r}=-\left\langle\zeta_{r}\left(\zeta^{2}-\frac{5}{2}\right) \phi\right\rangle \equiv-Q_{r}[\phi], \quad(|\boldsymbol{x}|=1) .
\end{gathered}
$$

Here, $\mathcal{L}[\phi]$ is the linearised collision integral (Sone 2007) whose explicit form is not required,

$$
g_{w}(\boldsymbol{\zeta})=2 \Omega \zeta_{\varphi} \sin \theta+\left(|\boldsymbol{\zeta}|^{2}-\frac{5}{2}\right) \tau_{s}
$$

and $\phi^{*}, g_{w}^{*}$ and $E^{*}$ are $\phi^{*}=\phi\left(\boldsymbol{x}, \boldsymbol{\zeta}^{*}\right), g_{w}^{*}=g_{w}\left(\boldsymbol{\zeta}^{*}\right)$ and $E\left(\boldsymbol{\zeta}^{*}\right)$, respectively. The function $g_{w}$ depends also on the position on the sphere through $\theta$ and $\tau_{s}$. Equation (A $2 b$ ), which states the continuity of heat flow across the surface, is the linearised version of the conservation of energy on the surface (i.e. the continuity of energy flow across the surface).

The operator $\mathcal{L}$ satisfies the following well-known properties:

(i)

$$
\mathcal{L}[\varphi]=0 \Longleftrightarrow \varphi(\boldsymbol{\zeta}) \text { is a linear combination of } 1, \boldsymbol{\zeta} \text { and }|\boldsymbol{\zeta}|^{2} \text {. }
$$

(ii) For any function $\varphi(\boldsymbol{\zeta})$,

$$
\langle\varphi \mathcal{L}[\varphi]\rangle \leqslant 0
$$

and the equality holds if and only if $\varphi$ is a linear combination of $1, \boldsymbol{\zeta}$ and $|\boldsymbol{\zeta}|^{2}$.

The scattering kernel $K_{B 0}\left(\boldsymbol{\zeta}, \boldsymbol{\zeta}^{*}\right)$ is required to satisfy the following basic properties. Let $n_{i}$ be the unit normal vector on the boundary pointing the the gas and let $\zeta_{n}=\zeta_{i} n_{i}$ and $\zeta_{n}^{*}=\zeta_{i}^{*} n_{i}$. Then,

(i) Positivity:

$$
K_{B 0}\left(\boldsymbol{\zeta}, \boldsymbol{\zeta}^{*}\right) \geqslant 0, \quad \text { for } \zeta_{n}^{*}<0 \text { and } \zeta_{n}>0
$$

(ii) Impermeability:

$$
\int_{\zeta_{n}>0} \frac{\zeta_{n}}{\zeta_{n}^{*}} K_{B 0}\left(\boldsymbol{\zeta}, \boldsymbol{\zeta}^{*}\right) \mathrm{d} \boldsymbol{\zeta}=-1, \quad \zeta_{n}^{*}<0
$$

(iii) Uniqueness: let $\varphi_{e}=c_{0}+c_{i} \zeta_{i}+c_{4}|\boldsymbol{\zeta}|^{2}$, where $c_{0}, c_{i}(i=1,2,3)$ and $c_{4}$ are independent of $\zeta$. Then, the equality

$$
\varphi_{e} E=\int_{\zeta_{n}^{*}<0} K_{B 0}\left(\boldsymbol{\zeta}, \boldsymbol{\zeta}_{*}\right) \varphi_{e}\left(\boldsymbol{\zeta}^{*}\right) E^{*} \mathrm{~d} \boldsymbol{\zeta}^{*}, \quad\left(\zeta_{n}>0\right),
$$

holds if and only if $c_{1}=c_{2}=c_{3}=c_{4}=0$.

Further, if $K_{B 0}\left(\boldsymbol{\zeta}, \boldsymbol{\zeta}^{*}\right)$ satisfies the above properties, the following inequality holds on the boundary: 
- Darrozes-Guiraud inequality (Cercignani 1988; Sone 2007): Let $F(x)$ be a strictly convex function. Then, for any $\varphi(\boldsymbol{\zeta})$ satisfying

$$
\varphi E=\int_{\zeta_{n}^{*}<0} K_{B 0}\left(\boldsymbol{\zeta}, \boldsymbol{\zeta}^{*}\right) \varphi\left(\boldsymbol{\zeta}^{*}\right) E^{*} \mathrm{~d} \boldsymbol{\zeta}^{*}, \quad\left(\zeta_{n}>0\right),
$$

the following inequality holds

$$
\left\langle\zeta_{n} F(\varphi)\right\rangle \leqslant 0
$$

The equality sign applies if and only if $\varphi$ is independent of $\boldsymbol{\zeta}$.

We first consider a reduced problem for $\phi$ derived from (A 1a)-(A 1c) by setting $\tau_{s} \equiv 0$ in the boundary condition (A $1 b)$, i.e. $g_{w} \equiv 2 \Omega \zeta_{\varphi} \sin \theta$. The solution to this problem is denoted by $\phi^{0}$. Clearly, this corresponds to the situation considered in the main text; the temperature of the sphere is uniform and coincides with that of the gas at infinity. If the scattering operator defined by the kernel $K_{B 0}\left(\boldsymbol{\zeta}, \boldsymbol{\zeta}^{*}\right)$ admits an axial symmetry about the axis normal to the boundary, the same similarity solution of the form (2.14) is applicable, and consequently, the heat flux across the boundary vanishes since $Q_{r}\left[\phi^{0}\right]=0$ [see the line following (2.20)]. Consequently, $\tau_{s}=0$ is a solution to the problem (A $2 a$ ) and $(\mathrm{A} 2 b)$ (with $\left.\phi=\phi^{0}\right)$. Thus, we conclude that $\left(\phi, \tau_{s}\right)=\left(\phi^{0}, 0\right)$ is a solution to the (full) boundary-value problem (A $1 a)-(\mathrm{A} 2 b)$. Moreover, the uniqueness of solution (see below) assures that $\left(\phi, \tau_{s}\right)=\left(\phi^{0}, 0\right)$ is the only solution to the problem (A $\left.1 a\right)-(\mathrm{A} 2 b)$.

The uniqueness of solution can be shown along the same line as that of the boundaryvalue problem of the linearised Boltzmann equation (without the stationary heatconduction equation) (see e.g. Sone 2007, A.12). However, the inclusion of the heatconduction equation results in an interesting application of the uniqueness condition for the scattering kernel, which is illustrative. Therefore, we present a proof here.

Our goal is to show that the solution to the problem (A 1a)-(A 1c) with $\Omega=0$ vanishes identically, i.e. $\left(\phi, \tau_{s}\right)=(0,0)$. To see this, we multiply the equation (A $\left.1 a\right)$ by $2 \phi E$ and integrate the result with respect to $\zeta_{i}$ over the whole space to obtain $\dagger$

$$
\frac{\partial}{\partial x_{i}}\left\langle\zeta_{i} \phi^{2}\right\rangle=\frac{2}{k}\langle\phi \mathcal{L}[\phi]\rangle \equiv g(\boldsymbol{x}) \leqslant 0
$$

Further integration with respect to $\boldsymbol{x}$ over the whole gas region gives

$$
\int_{|\boldsymbol{x}|>1} \frac{\partial}{\partial x_{i}}\left\langle\zeta_{i} \phi^{2}\right\rangle \mathrm{d} \boldsymbol{x}=\int_{|\boldsymbol{x}|>1} g(\boldsymbol{x}) \mathrm{d} \boldsymbol{x} \equiv \mathcal{G} \leqslant 0 .
$$

Applying Gauss's divergence theorem on the left-hand side yields

$$
\int_{|\boldsymbol{x}|>1} \frac{\partial}{\partial x_{i}}\left\langle\zeta_{i} \phi^{2}\right\rangle \mathrm{d} \boldsymbol{x}=\lim _{r \rightarrow \infty} \int_{|\boldsymbol{x}|=r}\left\langle\zeta_{r} \phi^{2}\right\rangle \mathrm{d} S-\int_{|\boldsymbol{x}|=1}\left\langle\zeta_{r} \phi^{2}\right\rangle \mathrm{d} S,
$$

where $\mathrm{d} S$ is the surface element. Noting that $\left\langle\zeta_{i} \phi^{2}\right\rangle=O\left(|\boldsymbol{x}|^{-3}\right)$ for $|\boldsymbol{x}| \gg 1 \ddagger$, the first term on the right-hand side vanishes and (A 12) reduces to

$$
-\int_{|\boldsymbol{x}|=1}\left\langle\zeta_{r} \phi^{2}\right\rangle \mathrm{d} S=\mathcal{G} \leqslant 0
$$

$\dagger$ Since the velocity distribution function contains discontinuities, the order of spatial derivative and integration cannot be interchanged freely. However, using the fact that the discontinuity of $\phi$ lies on the characteristics of the equation, one can show that the expression of the most left-hand side of (A 11) is legitimate (Sone 2007). The same note applies to the sentence containing (2.24).

$\ddagger$ This follows from the estimate $\phi=2 \zeta_{i} h_{i}+\left(|\boldsymbol{\zeta}|^{2}-5 / 2\right) h_{4}+O\left(|\boldsymbol{x}|^{-2}\right)$ for $|\boldsymbol{x}| \gg 1$, where $h_{i}$ $(i=1,2,3)$ and $h_{4}$, independent of $\boldsymbol{\zeta}$, are quantities of $O\left(|\boldsymbol{x}|^{-1}\right)$. 
Now if we put $F(x)=x^{2}, \varphi=\phi-\bar{g}_{w}$ and $\bar{g}_{w}=\left(|\boldsymbol{\zeta}|^{2}-5 / 2\right) \tau_{s}$ in the Darrozes-Guiraud inequality, the condition (A 9 ) is satisfied and we have

$$
\left\langle\zeta_{r}\left(\phi-\bar{g}_{w}\right)^{2}\right\rangle \leqslant 0, \quad(|\boldsymbol{x}|=1) .
$$

Since $\left\langle\zeta_{r} \bar{g}_{w}^{2}\right\rangle=0$, the left-hand side is transformed to

$$
\left\langle\zeta_{r}\left(\phi-\bar{g}_{w}\right)^{2}\right\rangle=\left\langle\zeta_{r} \phi^{2}\right\rangle-2\left\langle\zeta_{r} \bar{g}_{w} \phi\right\rangle=\left\langle\zeta_{r} \phi^{2}\right\rangle-2 \tau_{s} Q_{r}[\phi] \leqslant 0, \quad(|\boldsymbol{x}|=1) .
$$

Thus,

$$
\int_{|\boldsymbol{x}|=1}\left\langle\zeta_{r} \phi^{2}\right\rangle \mathrm{d} S \leqslant 2 \int_{|\boldsymbol{x}|=1} \tau_{s} Q_{r}[\phi] \mathrm{d} S .
$$

Combining this with (A 14), we obtain

$$
\int_{|\boldsymbol{x}|=1} \tau_{s} Q_{r}[\phi] \mathrm{d} S \geqslant 0 .
$$

On the other hand, multiplying (A $2 a$ ) by $\tau_{s}$ and integrating the result inside the sphere gives

$$
\int_{|\boldsymbol{x}|<1} \frac{\partial}{\partial x_{j}}\left(\tau_{s} \hat{\lambda}_{s} \frac{\partial \tau_{s}}{\partial x_{j}}\right) \mathrm{d} \boldsymbol{x}-\int_{|\boldsymbol{x}|<1} \hat{\lambda}_{s}\left(\frac{\partial \tau_{s}}{\partial x_{j}}\right)^{2} \mathrm{~d} \boldsymbol{x}=0 .
$$

Applying Gauss's divergence theorem to the first term gives

$$
\int_{|\boldsymbol{x}|<1} \frac{\partial}{\partial x_{j}}\left(\tau_{s} \hat{\lambda}_{s} \frac{\partial \tau_{s}}{\partial x_{j}}\right) \mathrm{d} \boldsymbol{x}=\int_{|\boldsymbol{x}|=1} \tau_{s} \hat{\lambda}_{s} \frac{\partial \tau_{s}}{\partial r} \mathrm{~d} S=-\int_{|\boldsymbol{x}|=1} \tau_{s} Q_{r}[\phi] \mathrm{d} S,
$$

where (A $2 b)$ has been used in the last equality. Therefore,

$$
\int_{|\boldsymbol{x}|=1} \tau_{s} Q_{r}[\phi] \mathrm{d} S=-\int_{|\boldsymbol{x}|<1} \hat{\lambda}_{s}\left(\frac{\partial \tau_{s}}{\partial x_{j}}\right)^{2} \mathrm{~d} \boldsymbol{x} \leqslant 0 .
$$

Hence, from (A 18) and (A 21), we have

$$
\int_{|\boldsymbol{x}|=1} \tau_{s} Q_{r}[\phi] \mathrm{d} S=\int_{|\boldsymbol{x}|<1} \hat{\lambda}_{s}\left(\frac{\partial \tau_{s}}{\partial x_{j}}\right)^{2} \mathrm{~d} \boldsymbol{x}=0 .
$$

Thus, $\tau_{s}$ is a constant independent of $\boldsymbol{x}$. Also from (A 14), (A 17) and (A 22),

$$
-\int_{|\boldsymbol{x}|=1}\left\langle\zeta_{r} \phi^{2}\right\rangle \mathrm{d} S=\mathcal{G}=\frac{2}{k} \int_{|\boldsymbol{x}|>1}\langle\phi \mathcal{L}[\phi]\rangle(\boldsymbol{x}) \mathrm{d} \boldsymbol{x}=0 .
$$

However, since $\langle\phi \mathcal{L}[\phi]\rangle$ is non-positive (see (A 5)), $\langle\phi \mathcal{L}[\phi]\rangle=0$ must hold throughout the gas region. This shows that $\phi$ is a linear combination of $1, \boldsymbol{\zeta}$ and $|\boldsymbol{\zeta}|^{2}$. As the result, $\mathcal{L}[\phi]=0$ holds and $(\mathrm{A} 1)$ reduces to

$$
\zeta_{i} \frac{\partial \phi}{\partial x_{i}}=0
$$

Therefore, $\phi$ is invariant along the characteristics, which implies that $\phi=0$ for $\zeta_{i}$ whose corresponding characteristic can be traced back to the infinity. In particular, $\phi=0$ for the incident molecules $\left(\zeta_{r}<0\right)$ on the boundary $(|\boldsymbol{x}|=1)$. With this, (A 23) is rewritten to give

$$
\int_{|\boldsymbol{x}|=1} \int_{\zeta_{r}>0} \zeta_{r} \phi^{2} E \mathrm{~d} \boldsymbol{\zeta} \mathrm{d} S=0
$$


which shows that $\phi=0$ holds also for the outgoing molecules $\zeta_{r}>0$ on the boundary $(|\boldsymbol{x}|=1)$. Thus, we can conclude that $\phi=0$ in the whole gas region. Then, (A 1b) (with $\Omega=0$ ) reduces to

$$
\bar{g}_{w} E=\int_{\zeta_{r}^{*}<0} K_{B 0}\left(\boldsymbol{\zeta}, \boldsymbol{\zeta}^{*}\right) \bar{g}_{w}^{*} E^{*} \mathrm{~d} \boldsymbol{\zeta}^{*}, \quad \zeta_{r}>0, \quad(|\boldsymbol{x}|=1) .
$$

However, due to the uniqueness condition for $K_{B 0}, \tau_{s}$ must vanish on the boundary. Thus, we conclude that $\tau_{s}=0$ also inside the sphere, since $\tau_{s}$ is constant.

One might think that the uniform temperature of the sphere and the gas (i.e. $\tau=\tau_{s}=$ 0 ) is physically obvious for the following reason. In a slow flow for which a linearisation is applicable, the energy equation of the gas reduces to $\partial Q_{i} / \partial x_{i}=0$, which is identical with the energy equation in a solid body. Consequently, in the absence of heat sources in the body and under the condition of energy continuity across the surface, the radial heat flux through the sphere and the gas is zero, which results in a uniform temperature of the sphere and the gas. However, this argument does not hold in rarefied gases, though the result is still true for the present rotating flow. The reasons are the followings. First, we cannot conclude that $\tau=$ const from $Q_{i}=0$, since the Fourier law does not hold generally in rarefied gases. In the present problem, $\tau \equiv 0$ is derived as a consequence of the similarity solution. Second, we cannot conclude that there is no radial heat flux in the sphere in the absence of heat sources inside when the ambient gas is a rarefied gas. In rarefied gases, the flow velocity and the heat flux are linked through the velocity distribution function, and if any nonzero radial heat flux is induced in the gas, it may cause a redistribution of the temperature (in a thermally neutral body), resulting in a nonuniform temperature distribution (thermal polarisation) $\dagger$. The present linearised steady rotating flow is free from this effect because it produces no radial heat flux in the gas (i.e. $\left.Q_{r} \equiv 0\right)$. Here, we stress again that the vanishing radial heat flux is a consequence of the similarity solution and is not derived from $\tau \equiv 0$. In rarefied gases, $\tau=$ const and $Q_{i}=0$ are not equivalent. Indeed, $Q_{\varphi} \neq 0$ despite $\tau \equiv 0$ in the present rotating flow. Incidentally, the thermal polarisation of a sphere has been extensively studied in the literature (see e.g. Beresnev et al. 1990; Takata \& Sone 1995, and the references therein).

A steady nonuniform temperature distribution of the sphere and the surrounding gas without total heat production in a thermally adiabatic system does not conflict with the thermodynamic laws when there are inputs of energy (i.e. work) per unit time. If this work is solely associated with the sphere rotation, the problem separation and the uniqueness result exclude such a possibility in the linearised framework.

\section{Appendix B. Estimate of the first term on the right-hand side of (6.2)}

In this appendix, we estimate the behaviour of the first term of the right-hand side of (6.2). To this end, we follow the basic strategy in Takata \& Taguchi (2017) and summarise the main points.

Let us first consider the so-called partial model, obtained by omitting the gain term of the linearised ES equation (or the linearised Boltzmann equation):

$$
\zeta_{i} \frac{\partial \phi}{\partial x_{i}}=-\frac{\nu_{c}}{k} \phi
$$

which is supplemented by the boundary conditions (2.6) and (2.7). Here, $\nu_{c}=\nu_{c}(\zeta)>$ $\delta(>0)$ with $\delta$ being a positive constant $\left(\nu_{c}=1\right.$ in the case of the linearised ES model).

$\dagger$ This in turn affects the flow around the sphere. 
After applying the form $\phi=\Omega \zeta_{\varphi} \phi_{S}\left(r, \theta_{\zeta}, \zeta\right) \sin \theta$, the solution $\phi_{S}$ for the partial model is given by

$$
\begin{aligned}
\phi_{S}\left(r, \theta_{\zeta}, \zeta\right) & = \begin{cases}2 \alpha r \exp \left(-\frac{\nu_{c} \sigma_{B}\left(r, \theta_{\zeta}\right)}{k \zeta}\right), & {\left[0 \leqslant \theta_{\zeta} \leqslant \operatorname{Arcsin}\left(r^{-1}\right)\right],} \\
0, & {\left[\operatorname{Arcsin}\left(r^{-1}\right)<\theta_{\zeta} \leqslant \pi\right],}\end{cases} \\
\sigma_{B}\left(r, \theta_{\zeta}\right) & =r \cos \theta_{\zeta}-\left(1-r^{2} \sin ^{2} \theta_{\zeta}\right)^{1 / 2} .
\end{aligned}
$$

Thus, in the case of the partial model, it is sufficient to consider the case of $\alpha=1$. Keeping this in mind, we evaluate the first term of (6.2) as follows:

$$
\begin{aligned}
\text { (first term) } & =\pi \int_{0}^{\infty} \int_{0}^{\operatorname{Arcsin}\left(\frac{1}{r}\right)} \zeta^{4} \sin ^{3} \theta_{\zeta} \frac{\partial \phi_{S}}{\partial r}\left(r, \theta_{\zeta}, \zeta\right) E \mathrm{~d} \theta_{\zeta} \mathrm{d} \zeta \\
& =2 \pi \int_{0}^{\infty} \int_{0}^{\operatorname{Arcsin}\left(\frac{1}{r}\right)} \zeta^{4} \sin ^{3} \theta_{\zeta} \frac{\partial}{\partial r}\left[r \exp \left(-\frac{\nu_{c} \sigma_{B}\left(r, \theta_{\zeta}\right)}{k \zeta}\right)\right] E \mathrm{~d} \theta_{\zeta} \mathrm{d} \zeta \\
& =2 \pi \int_{0}^{\infty} \int_{0}^{\operatorname{Arcsin}\left(\frac{1}{r}\right)} \zeta^{4} \sin ^{3} \theta_{\zeta}\left(1-\frac{\nu_{c} r}{k \zeta} \cos \theta_{\zeta}\right) \exp \left(-\frac{\nu_{c} \sigma_{B}\left(r, \theta_{\zeta}\right)}{k \zeta}\right) E \mathrm{~d} \theta_{\zeta} \mathrm{d} \zeta \\
& -\frac{2 \pi}{k} \int_{0}^{\infty} \int_{0}^{\operatorname{Arcsin}\left(\frac{1}{r}\right)} \frac{\nu_{c} r^{2} \zeta^{3} \sin ^{5} \theta_{\zeta}}{\left(1-r^{2} \sin ^{2} \theta_{\zeta}\right)^{1 / 2}} \exp \left(-\frac{\nu_{c} \sigma_{B}\left(r, \theta_{\zeta}\right)}{k \zeta}\right) E \mathrm{~d} \theta_{\zeta} \mathrm{d} \zeta .
\end{aligned}
$$

The first term obviously remains finite as $r \downarrow 1$. On the other hand, the second term is estimated as

$$
\begin{aligned}
& \left|\int_{0}^{\infty} \int_{0}^{\operatorname{Arcsin}\left(\frac{1}{r}\right)} \frac{\nu_{c} r^{2} \zeta^{3} \sin ^{5} \theta_{\zeta}}{\left(1-r^{2} \sin ^{2} \theta_{\zeta}\right)^{1 / 2}} \exp \left(-\frac{\nu_{c} \sigma_{B}\left(r, \theta_{\zeta}\right)}{k \zeta}\right) E \mathrm{~d} \theta_{\zeta} \mathrm{d} \zeta\right| \\
& \leqslant \int_{0}^{\infty} \int_{0}^{\operatorname{Arcsin}\left(\frac{1}{r}\right)} \frac{\nu_{c} r^{2} \zeta^{3}}{\left(1-r^{2} \sin ^{2} \theta_{\zeta}\right)^{1 / 2}} E \mathrm{~d} \theta_{\zeta} \mathrm{d} \zeta \\
& =\int_{0}^{\infty} \nu_{c} \zeta^{3} E \mathrm{~d} \zeta \int_{0}^{\pi / 2} \frac{r^{2}}{\left(r^{2}-\sin ^{2} \theta_{\zeta 0}\right)^{1 / 2}} \mathrm{~d} \theta_{\zeta 0}=\left(\int_{0}^{\infty} \nu_{c} \zeta^{3} E \mathrm{~d} \zeta\right) r K\left(\frac{1}{r}\right),
\end{aligned}
$$

where $K(x)$ is the complete elliptic integral of the first kind. Since $K(x) \sim \frac{1}{2} \ln \frac{16}{1-x^{2}}$ as $x \uparrow 1$, we find that

$$
r K\left(\frac{1}{r}\right) \sim \frac{1}{2} \ln \left(\frac{1}{r-1}\right)
$$

as $r \downarrow 1$. Thus, the second term diverges at most logarithmically. Also, notice that this logarithmic divergence of the first term of (6.2) does not occur when the gas is collisionless $(k=\infty)$, because the second term of (B 4) degenerates in this case.

Now we consider the following quasi-full model:

$$
\zeta_{i} \frac{\partial \phi}{\partial x_{i}}=-\frac{\nu_{c}}{k} \phi+\frac{S}{k},
$$

supplemented by the same boundary conditions as before, i.e. (2.6) and (2.7). Here, $S \equiv \Omega \zeta_{\varphi} \sin \theta S_{1}\left(r, \theta_{\zeta}, \zeta\right)$ represents a source term which is supposed to behave in the same way as the moments of $\phi$ of the partial model as $r \downarrow 1$. The ES model obviously satisfies this property. That is, $S_{1} \sim a\left(\theta_{\zeta}, \zeta\right)+b\left(\theta_{\zeta}, \zeta\right) s^{1 / 2}+c\left(\theta_{\zeta}, \zeta\right) s \ln s+\cdots$ with $s=r-1$. The second term proportional to $s^{1 / 2}$ is due to the contribution of the second term of (6.2).

Integrating the equation along the characteristics, the solution $\phi_{S}$ of $\phi=$ 
$\Omega \zeta_{\varphi} \phi_{S}\left(r, \theta_{\zeta}, \zeta\right) \sin \theta$ for the quasi-full model is given, for $\theta_{\zeta} \in\left[0, \operatorname{Arcsin}\left(r^{-1}\right)\right)$, as follows:

$$
\phi_{S}\left(r, \theta_{\zeta}, \zeta\right)=r \phi_{S}\left(1, \theta_{\zeta 0}, \zeta\right) \exp \left(-\frac{\nu_{c} \sigma_{B}}{k \zeta}\right)+\frac{1}{k} \int_{0}^{\sigma_{B}} \frac{r S_{1}\left(\widetilde{r}, \widetilde{\theta}_{\zeta}, \zeta\right)}{\zeta \widetilde{r}} \exp \left(-\frac{\nu_{c}\left(\sigma_{B}-t\right)}{k \zeta}\right) \mathrm{d} t .
$$

Here, $\sigma_{B}=\sigma_{B}\left(r, \theta_{\zeta}\right)$ is given by (B 3) and

$$
\begin{aligned}
& \theta_{\zeta 0}=\operatorname{Arcsin}\left(r \sin \theta_{\zeta}\right), \\
& \widetilde{r}=\left(t^{2}+2 t \cos \theta_{\zeta 0}+1\right)^{1 / 2}, \\
& \tilde{\theta}_{\zeta}=\operatorname{Arcsin}\left(\frac{r \sin \theta_{\zeta}}{\widetilde{r}}\right), \\
& \phi_{S}\left(1, \theta_{\zeta}, \zeta\right)=2 \alpha+(1-\alpha) \phi_{S}\left(1, \pi-\theta_{\zeta}, \zeta\right), \quad\left(0 \leqslant \theta_{\zeta} \leqslant \pi / 2\right) .
\end{aligned}
$$

Note that $\phi_{S}\left(1, \theta_{\zeta 0}, \zeta\right)$ in the first term depends on VDF of the incoming molecules on the boundary when $\alpha \neq 1$.

In order to verify that the inclusion of the source term does not change the behaviour of the moment of the partial model, we go back to the first term of (6.2) and consider the following partial integral:

$$
I=\pi \int_{0}^{\infty} \int_{0}^{\operatorname{Arcsin}\left(\frac{1}{r}\right)} \zeta^{4} \sin ^{3} \theta_{\zeta} \frac{\partial \phi_{S}}{\partial r}\left(r, \theta_{\zeta}, \zeta\right) E \mathrm{~d} \theta_{\zeta} \mathrm{d} \zeta
$$

Substituting $\phi_{S}$ into this expression, we obtain, after some manipulations,

$$
\begin{aligned}
& I= \pi \int_{0}^{\infty} \int_{0}^{\operatorname{Arcsin}\left(\frac{1}{r}\right)} \zeta^{4} \sin ^{3} \theta_{\zeta} \\
& \times\left[\left(1-\frac{\nu_{c} r \cos \theta_{\zeta}}{k \zeta}\right) \phi_{S}\left(1, \theta_{\zeta 0}, \zeta\right)+\frac{r \cos \theta_{\zeta}}{k \zeta} S_{1}\left(1, \theta_{\zeta 0}, \zeta\right)\right] \exp \left(-\frac{\nu_{c} \sigma_{B}}{k \zeta}\right) E \mathrm{~d} \theta_{\zeta} \mathrm{d} \zeta \\
&- \frac{\pi}{k} \int_{0}^{\infty} \int_{0}^{\operatorname{Arcsin}\left(\frac{1}{r}\right)} \frac{r^{2} \zeta^{3} \sin ^{5} \theta_{\zeta}}{\left(1-r^{2} \sin ^{2} \theta_{\zeta}\right)^{1 / 2}}\left[\nu_{c} \phi_{S}\left(1, \theta_{\zeta 0}, \zeta\right)-S_{1}\left(1, \theta_{\zeta 0}, \zeta\right)\right. \\
&+\left.\quad-\frac{k(1-\alpha) \zeta}{r} \frac{\partial \phi_{S}\left(1, \pi-\theta_{\zeta 0}, \zeta\right)}{\partial \theta_{\zeta 0}}\right] \exp \left(-\frac{\nu_{c} \sigma_{B}}{k \zeta}\right) E \mathrm{~d} \theta_{\zeta} \mathrm{d} \zeta \\
& \int_{0}^{\infty} \int_{0}^{\operatorname{Arcsin}\left(\frac{1}{r}\right)} \zeta^{3} \sin ^{3} \theta_{\zeta} \int_{0}^{\sigma_{B}} \mathcal{D}\left(\frac{r S_{1}\left(\widetilde{r}, \widetilde{\theta}_{\zeta}, \zeta\right)}{\widetilde{r}}\right) \exp \left(-\frac{\nu_{c}\left(\sigma_{B}-t\right)}{k \zeta}\right) \mathrm{d} t E \mathrm{~d} \theta_{\zeta} \mathrm{d} \zeta
\end{aligned}
$$

where

$$
\mathcal{D} g(t, r)=\left(\frac{\partial}{\partial r}+\frac{\partial \sigma_{B}}{\partial r} \frac{\partial}{\partial t}\right) g(t, r)
$$

Note that (B 4) for the partial model is recovered by setting $\phi_{S}\left(1, \theta_{\zeta}, \zeta\right)=2\left(0 \leqslant \theta_{\zeta} \leqslant\right.$ $\pi / 2)$ and $S_{1}=0$ as well as $\alpha=1$. Clearly, the first term remains finite as $r \downarrow 1$ if $\left|\phi_{S}\left(1, \theta_{\zeta}, \zeta\right)\right|$ is bounded for the impinging molecules $\theta_{\zeta} \in(\pi / 2, \pi]$. The second term involves the derivative of $\phi_{S}$ with respect to $\theta_{\zeta}$ on the boundary for the impinging molecules, $\theta_{\zeta} \in(\pi / 2, \pi]$. We will see later that this remains finite. Therefore, essentially the same estimate as in the case of the partial model applies and the integral is estimated 
to be logarithmically diverging as $r \downarrow 1$. For the third term, we first note that

$$
\begin{aligned}
& \mathcal{D} r=1, \\
& \mathcal{D} \widetilde{r}=\frac{\left(t+\sqrt{1-r^{2} \sin ^{2} \theta_{\zeta}}\right) \cos \theta_{\zeta}+r \sin ^{2} \theta_{\zeta}}{\widetilde{r}}, \\
& \mathcal{D} \tilde{\theta}_{\zeta}=\frac{\sin \theta_{\zeta}\left(t+\sqrt{1-r^{2} \sin ^{2} \theta_{\zeta}}-r \cos \theta_{\zeta}\right)}{\widetilde{r}^{2}} .
\end{aligned}
$$

Then, since $\left|\partial S_{1} / \partial r\right| \sim C / \sqrt{r-1}$ for some positive constant $C$ as $r \downarrow 1$, we have the following estimate: $\dagger$

$$
\left|\mathcal{D}\left(\frac{r S_{1}\left(\widetilde{r}, \tilde{\theta}_{\zeta}, \zeta\right)}{\widetilde{r}}\right)\right| \lesssim \frac{C r^{2}}{\sqrt{\widetilde{r}-1}}, \quad(\widetilde{r} \downarrow 1)
$$

Thus, we are left to examine the integral

$$
J=\frac{\pi}{k} \int_{0}^{\infty} \int_{0}^{\operatorname{Arcsin}\left(\frac{1}{r}\right)} \zeta^{3} \sin ^{3} \theta_{\zeta} \int_{0}^{\sigma_{B}} \frac{r^{2}}{(\widetilde{r}-1)^{1 / 2}} \exp \left(-\frac{\nu_{c}\left(\sigma_{B}-t\right)}{k \zeta}\right) \mathrm{d} t E \mathrm{~d} \theta_{\zeta} \mathrm{d} \zeta
$$

Since

$$
\begin{aligned}
\frac{1}{\sqrt{\widetilde{r}-1}} & =\frac{\sqrt{\widetilde{r}+1}}{\sqrt{\widetilde{r}^{2}-1}}=\frac{\sqrt{\widetilde{r}+1}}{\sqrt{t^{2}+2 t \cos \theta_{\zeta 0}}} \leqslant \frac{\sqrt{r+1}}{t^{1 / 2} \sqrt{t+2 \cos \theta_{\zeta 0}}} \\
& \leqslant \frac{\sqrt{r+1}}{t^{1 / 2} \sqrt{2 \cos \theta_{\zeta 0}}} \leqslant \frac{\sqrt{2} r}{t^{1 / 2} \sqrt{2 \cos \theta_{\zeta 0}}}=\frac{r}{t^{1 / 2} \sqrt{\cos \theta_{\zeta 0}}}
\end{aligned}
$$

we have

$$
\begin{aligned}
J & \leqslant \frac{\pi}{k} \int_{0}^{\infty} \int_{0}^{\operatorname{Arcsin}\left(\frac{1}{r}\right)} \zeta^{3} r^{3} \sin ^{3} \theta_{\zeta} \frac{1}{\sqrt{\cos \theta_{\zeta 0}}} \int_{0}^{\sigma_{B}} \frac{\mathrm{d} t}{t^{1 / 2}} E \mathrm{~d} \theta_{\zeta} \mathrm{d} \zeta \\
& \leqslant \frac{2 \pi}{k} \int_{0}^{\infty} \int_{0}^{\operatorname{Arcsin}\left(\frac{1}{r}\right)} \zeta^{3} r^{3} \sin ^{3} \theta_{\zeta} \frac{\sqrt{\sigma_{B}}}{\sqrt{\cos \theta_{\zeta 0}}} E \mathrm{~d} \theta_{\zeta} \mathrm{d} \zeta \\
& =\frac{2 \pi}{k} \int_{0}^{\infty} \zeta^{3} E \mathrm{~d} \zeta \int_{0}^{\pi / 2} \sin ^{3} \theta_{\zeta 0} \frac{\sqrt{\sigma_{B}}}{\sqrt{\cos \theta_{\zeta 0}}} \frac{\cos \theta_{\zeta 0}}{\sqrt{r^{2}-\sin ^{2} \theta_{\zeta 0}}} \mathrm{~d} \theta_{\zeta 0} .
\end{aligned}
$$

But, since

$$
\sigma_{B}=r \cos \theta_{\zeta}-\sqrt{1-r^{2} \sin ^{2} \theta_{\zeta}} \leqslant r \cos \theta_{\zeta}=r \sqrt{1-\sin ^{2} \theta_{\zeta}}=\sqrt{r^{2}-\sin ^{2} \theta_{\zeta 0}}
$$

$\dagger$ Since $t \leqslant \sigma_{B}$ in the range of integration, $\mathcal{D} \widetilde{r} \leqslant \frac{\left(\sigma_{B}+\sqrt{1-r^{2} \sin ^{2} \theta_{\zeta}}\right) \cos \theta_{\zeta}+r \sin ^{2} \theta_{\zeta}}{\widetilde{r}}=\frac{r \cos ^{2} \theta_{\zeta}+r \sin ^{2} \theta_{\zeta}}{r}=\frac{r}{\widetilde{r}}$. 


$$
\begin{aligned}
J & \leqslant \frac{2 \pi}{k} \int_{0}^{\infty} \zeta^{3} E \mathrm{~d} \zeta \int_{0}^{\pi / 2} \sin ^{3} \theta_{\zeta 0} \frac{\left(r^{2}-\sin ^{2} \theta_{\zeta 0}\right)^{1 / 4}}{\sqrt{\cos \theta_{\zeta 0}}} \frac{\cos \theta_{\zeta 0}}{\sqrt{r^{2}-\sin ^{2} \theta_{\zeta 0}}} \mathrm{~d} \theta_{\zeta 0} \\
& =\frac{2 \pi}{k} \int_{0}^{\infty} \zeta^{3} E \mathrm{~d} \zeta \int_{0}^{\pi / 2} \frac{\sin ^{3} \theta_{\zeta 0}}{\sqrt{\cos \theta_{\zeta 0}}} \frac{\cos \theta_{\zeta 0}}{\left(r^{2}-\sin ^{2} \theta_{\zeta 0}\right)^{1 / 4}} \mathrm{~d} \theta_{\zeta 0} \\
& \leqslant \frac{2 \pi}{k} \int_{0}^{\infty} \zeta^{3} E \mathrm{~d} \zeta \int_{0}^{\pi / 2} \frac{1}{\sqrt{\cos \theta_{\zeta 0}}} \frac{\cos \theta_{\zeta 0}}{\left(1-\sin ^{2} \theta_{\zeta 0}\right)^{1 / 4}} \mathrm{~d} \theta_{\zeta 0} \\
& =\frac{2 \pi}{k} \int_{0}^{\infty} \zeta^{3} E \mathrm{~d} \zeta \int_{0}^{\pi / 2} \mathrm{~d} \theta_{\zeta 0}=\frac{\pi^{2}}{k} \int_{0}^{\infty} \zeta^{3} E \mathrm{~d} \zeta .
\end{aligned}
$$

Thus, the third term is also bounded.

We close this appendix by showing the boundedness of $\left|\partial \phi_{S} / \partial \theta_{\zeta}\right|$ at $r=1$ for $\theta_{\zeta} \in$ $(\pi / 2, \pi]$. This is directly seen by writing $\phi_{S}\left(1, \theta_{\zeta}, \zeta\right)$ for $\pi / 2 \leqslant \theta_{\zeta} \leqslant \pi$ as (see (B 8))

$$
\begin{aligned}
& \phi_{S}\left(1, \theta_{\zeta}, \zeta\right)=\frac{1}{k} \int_{0}^{\infty} \frac{1}{\zeta} \frac{S_{1}\left(\widetilde{r}, \tilde{\theta}_{\zeta}, \zeta\right)}{\widetilde{r}} \exp \left(-\frac{\nu_{c} t}{k \zeta}\right) \mathrm{d} t, \quad\left(\frac{\pi}{2} \leqslant \theta_{\zeta} \leqslant \pi\right), \\
& \widetilde{r}=\left(t^{2}-2 t \cos \theta_{\zeta}+1\right)^{1 / 2}, \\
& \widetilde{r} \sin \widetilde{\theta}_{\zeta}=\sin \theta_{\zeta}, \quad\left(\pi / 2 \leqslant \widetilde{\theta}_{\zeta} \leqslant \pi\right) .
\end{aligned}
$$

Differentiating (B 15a) with respect to $\theta_{\zeta}$, we have

$$
\frac{\partial \phi_{S}\left(1, \theta_{\zeta}, \zeta\right)}{\partial \theta_{\zeta}}=\frac{1}{k} \int_{0}^{\infty} \frac{1}{\zeta} \frac{\partial}{\partial \theta_{\zeta}}\left[\frac{S_{1}\left(\widetilde{r}, \widetilde{\theta}_{\zeta}, \zeta\right)}{\widetilde{r}}\right] \exp \left(-\frac{\nu_{c} t}{k \zeta}\right) \mathrm{d} t
$$

where

$$
\begin{gathered}
\frac{\partial}{\partial \theta_{\zeta}}\left[\frac{S_{1}\left(\widetilde{r}, \widetilde{\theta}_{\zeta}, \zeta\right)}{\widetilde{r}}\right]=\left(-\frac{S_{1}\left(\widetilde{r}, \widetilde{\theta}_{\zeta}, \zeta\right)}{\widetilde{r}^{2}}+\frac{1}{\widetilde{r}} \frac{\partial S_{1}\left(\widetilde{r}, \widetilde{\theta}_{\zeta}, \zeta\right)}{\partial \widetilde{r}}\right) \frac{\partial \widetilde{r}}{\partial \theta_{\zeta}}+\frac{1}{\widetilde{r}} \frac{\partial S_{1}\left(\widetilde{r}, \widetilde{\theta}_{\zeta}, \zeta\right)}{\partial \widetilde{\theta}_{\zeta}} \frac{\partial \widetilde{\theta}_{\zeta}}{\partial \theta_{\zeta}}, \\
\frac{\partial \widetilde{r}}{\partial \theta_{\zeta}}=\frac{t}{\widetilde{r}} \sin \theta_{\zeta}, \quad \frac{\partial \widetilde{\theta}_{\zeta}}{\partial \theta_{\zeta}}=\frac{t \cos \theta_{\zeta}-1}{\widetilde{r}^{2}} .
\end{gathered}
$$

Thus, recalling again that $\left|\partial S_{1} / \partial r\right| \sim C / \sqrt{r-1}$ as $r \downarrow 1$, we have the estimate

$$
\left|\frac{\partial}{\partial \theta_{\zeta}}\left[\frac{S_{1}\left(\widetilde{r}, \widetilde{\theta}_{\zeta}, \zeta\right)}{\widetilde{r}}\right]\right| \lesssim \frac{C t}{\widetilde{r}^{2} \sqrt{\widetilde{r}-1}},
$$

uniformly in $\theta_{\zeta} \in(\pi / 2, \pi]$. In view of this, we consider the integral

$$
\frac{1}{k} \int_{0}^{\infty} \frac{1}{\zeta} \frac{t}{\widetilde{r}^{2} \sqrt{\widetilde{r}-1}} \exp \left(-\frac{\nu_{c} t}{k \zeta}\right) \mathrm{d} t
$$

Since

$$
\begin{gathered}
\frac{t}{\sqrt{\widetilde{r}-1}}=\frac{t \sqrt{\widetilde{r}+1}}{\sqrt{\widetilde{r}^{2}-1}}=\frac{t \sqrt{\widetilde{r}+1}}{\sqrt{t^{2}-2 t \cos \theta_{\zeta}}} \leqslant \frac{t \sqrt{\widetilde{r}+1}}{\sqrt{t^{2}}}=\sqrt{\widetilde{r}+1} \leqslant \sqrt{2} \widetilde{r}^{2}, \\
\frac{1}{k} \int_{0}^{\infty} \frac{1}{\zeta} \frac{t}{\widetilde{r}^{2} \sqrt{\widetilde{r}-1}} \exp \left(-\frac{\nu_{c} t}{k \zeta}\right) \mathrm{d} t \leqslant \frac{\sqrt{2}}{k \zeta} \int_{0}^{\infty} \exp \left(-\frac{\nu_{c} t}{k \zeta}\right) \mathrm{d} t=\frac{\sqrt{2}}{\nu_{c}} .
\end{gathered}
$$

Thus, $\left|\partial \phi_{S}\left(1, \theta_{\zeta}, \zeta\right) / \partial \theta_{\zeta}\right|$ is bounded for $\theta_{\zeta} \in(\pi / 2, \pi]$. 


\section{REFERENCES}

Andries, P., Tallec, P. Le, Perlat, J.-P. \& Perthame, B. 2000 The Gaussian-BGK model of Boltzmann equation with small Prandtl number. Eur. J. Mech. B/Fluids 19 (6), 813830.

Beresnev, S. A., Chernyak, V. G. \& Fomyagin, G. A. 1990 Motion of a spherical particle in a rarefied gas. Part 2. Drag and thermal polarization. J. Fluid Mech. 219, 405-421.

Bhatnagar, P. L., Gross, E. P. \& Krook, M. 1954 A model for collision processes in gases. I. Small amplitude processes in charged and neutral one-component systems. Phys. Rev. 94, 511-525.

Brult, S. 2015 An ellipsoidal statistical model for gas mixtures. Commun. Math. Sci. 13 (1), $1-13$.

Cercignani, C. 1988 The Boltzmann Equation and Its Applications. Berlin: Springer-Verlag.

Holway, J. 1966 New statistical models for kinetic theory: Methods of construction. Phys. Fluids 9 (9), 1658-1673.

Loyalka, S.K. \& Hickey, K.A. 1989 Plane Poiseuille flow: Near continuum results for a rigid sphere gas. Physica A 160 (3), 395-408.

LoYAlKA, S. K. 1992 Motion of a sphere in a gas: Numerical solution of the linearized Boltzmann equation. Phys. Fluids A 4 (5), 1049-1056.

Rubinow, S. I. \& Keller, Joseph B. 1961 The transverse force on a spinning sphere moving in a viscous fluid. J. Fluid Mech. 11, 447-459.

Sone, Y. 1973 New kind of boundary layer over a convex solid boundary in a rarefied gas. Phys. Fluids 16 (9), 1422-1424.

Sone, Y. 2002 Kinetic Theory and Fluid Dynamics. Boston: Birkhäuser, Supplementary Notes and Errata: Kyoto University Research Information Repository (http://hdl.handle.net/2433/66099).

Sone, Y. 2007 Molecular Gas Dynamics: Theory, Techniques, and Applications. Boston: Birkhäuser, Supplementary Notes and Errata: Kyoto University Research Information Repository (http://hdl.handle.net/2433/66098).

Sone, Y. \& TAKata, S. 1992 Discontinuity of the velocity distribution function in a rarefied gas around a convex body and the $\mathrm{S}$ layer at the bottom of the Knudsen layer. Transp. Theory Stat. Phys. 21, 501-530.

Sugimoto, H. \& Sone, Y. 1992 Numerical analysis of steady flows of a gas evaporating from its cylindrical condensed phase on the basis of kinetic theory. Phys. Fluids A 4, 419-440.

TAGuchi, S. 2015 Asymptotic theory of a uniform flow of a rarefied gas past a sphere at low Mach numbers. J. Fluid Mech. 774, 363-394.

TAguchi, S. \& Suzuki, T. 2017 Asymptotic far-field behavior of macroscopic quantities in a problem of slow uniform rarefied gas flow past a sphere. Phys. Rev. Fluids 2, 113401.

TAkata, S. \& Funagane, H. 2011 Poiseuille and thermal transpiration flows of a highly rarefied gas: Over-concentration in the velocity distribution function. J. Fluid Mech. 669, 242-259.

Takata, S., Hattori, M. \& Hasebe, T. $2016 a$ Slip/jump coefficients and Knudsen-layer corrections for the ES model in the generalized slip-flow theory. AIP Conf. Proc. 1786 (1), 040004 .

TAKata, S. \& Sone, Y. 1995 Flow induced around a sphere with a non-uniform surface temperature in a rarefied gas, with application to the drag and thermal force problems of a spherical particle with an arbitrary thermal conductivity. Eur J. Mech. B/Fluids 14, $487-518$.

TAkata, S., Sone, Y. \& Aoki, K. 1993 Numerical analysis of a uniform flow of a rarefied gas past a sphere on the basis of the Boltzmann equation for hard-sphere molecules. Phys. Fluids A 5 (3), 716-737.

TAKatA, S. \& TAguchi, S. 2017 Gradient divergence of fluid-dynamic quantities in rarefied gases on smooth boundaries. J. Stat. Phys. 168 (6), 1319-1352.

Takata, S., Yoshida, T., Noguchi, T. \& Taguchi, S. $2016 b$ Singular behavior of the macroscopic quantities in the free molecular gas. Phys. Fluids 28 (2), 022002.

Wakabayashi, M., Ohwada, T. \& Golse, F. 1996 Numerical analysis of the shear and thermal creep flows of a rarefied gas over the plane wall of a Maxwell-type boundary on the basis of the linearized Boltzmann equation for hard-sphere molecules. Eur. J. Mech. B/Fluids 15, 175-201. 
Welander, P. 1954 On the temperature jump in a rarefied gas. Ark. Fys. 7, 507-553. 